\title{
Distribution of refractory and volatile elements in CoRoT exoplanet host stars ${ }^{\star}$
}

\author{
C. Chavero ${ }^{1}$, R. de la Reza ${ }^{1}$, R. C. Domingos ${ }^{3}$, N. A. Drake ${ }^{2}$, C. B. Pereira ${ }^{1}$, and O. C. Winter $^{3}$ \\ 1 Observatório Nacional, Rua José Cristino 77, São Cristovão, 20921-400 Rio de Janeiro, Brazil \\ e-mail: [carolina; delareza; drake;claudio]@on.br \\ 2 Sobolev Astronomical Institute, St. Petersburg State University, Universitetski pr. 28, St. Petersburg 198504, Russia \\ 3 Univ Estadual Paulista - UNESP, Grupo de Dinâmica Orbital \& Planetologia, Guaratinguetá, CEP 12.516-410, Brazil \\ e-mail: rcassia@feg.unesp.br; ocwinter@pq.cnpq.br
}

Received 25 March 2009 / Accepted 29 March 2010

\section{ABSTRACT}

\begin{abstract}
The relative distribution of abundances of refractory, intermediate, and volatile elements in stars with planets can be an important tool for investigating the internal migration of a giant planet. This migration can lead to the accretion of planetesimals and the selective enrichment of the star with these elements. We report on a spectroscopic determination of the atmospheric parameters and chemical abundances of the parent stars in transiting planets CoRoT-2b and CoRoT-4b. Adding data for CoRoT-3 and CoRoT-5 from the literature, we find a flat distribution of the relative abundances as a function of their condensation temperatures. For CoRoT-2, the relatively high lithium abundance and intensity of its Li I resonance line permit us to propose an age of 120 Myr, making this star one of the youngest stars with planets to date. We introduce a new methodology to investigate a relation between the abundances of these stars and the internal migration of their planets. By simulating the internal migration of a planet in a disk formed only by planetesimals, we are able, for the first time, to separate the stellar fractions of refractory, intermediate, and volatile rich planetesimals accreting onto the central star. Intermediate and volatile element fractions enriching the star are similar and much larger than those of pure refractory ones. This result is opposite to what has been considered in the literature for the accreting self-enrichment processes of stars with planets. We also show that these results are highly dependent on the model adopted for the disk distribution regions in terms of refractory, intermediate, and also volatile elements and other parameters considered. We note however, that this self-enrichment mechanism is only efficient during the first 20-30 Myr or later in the lifetime of the disk when the surface convection layers of the central star for the first time attain its minimum size configuration.
\end{abstract}

Key words. planetary systems - stars: abundances - stars: fundamental parameters

\section{Introduction}

Gonzalez (1997) and Santos et al. (2001) first identified a metallicity excess in stars with giant planets (SWP) relative to stars without planets. The tendency has been confirmed using large samples of stars. Nevertheless, planets have also been observed around low metallicity main-sequence stars (Cochran et al. 2007). We note, however, that such a large statistical metal excess found among dwarf stars is not present in giant stars with planets (Pasquini et al. 2007).

Using iron as a primary reference element, this metal excess, has been the source of a number of studies trying to explain this property. Two scenarios have been invoked: a self-enrichment mechanism of normal metal stars and a primordial scenario, in which SWP are the result of the formation of entire metal-rich stars in equally metal-rich natal clouds. Which scenario is correct remains an open question to this day.

What about CoRoT exoplanet host stars? Although there are few cases with which to perform a statistical study, these stars exhibit a different distributions: the majority have solar abundances, one has a mild metal excess, and one has a low metal abundance. Even at this early stage, we are tempted to speculate that CoRoT is exploring a different backyard of the Galaxy with different properties from those of the Solar vicinity. Where

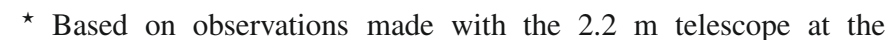
European Southern Observatory (La Silla, Chile). does the bulk of the metal excess of the SWP mentioned above originate?

The relative distribution of the stellar abundances of refractory elements, with a high condensation temperature $\left(T_{\mathrm{C}}\right)$, with respect to the volatile elements (low $T_{\mathrm{C}}$ ), has been widely used in the literature as a tool to investigate the nature of metal enrichment in SWP (see review papers of Gonzalez 2003, 2006b).

This enrichment mechanism should be triggered by the action of a hot Jupiter. The observed pile up of hot Jupiters is believed to be the result of migration. These planets formed further away in the protoplanetary nebula and migrated afterwards to the small orbital distances at which they are observed. During inward migration, material (planetesimals) from the disk, depleted in $\mathrm{H}$ and $\mathrm{He}$, could be accreted onto the star. The presence of a positive slope of $[\mathrm{X} / \mathrm{H}]$ versus $T_{\mathrm{C}}$, in which the abundances of volatile elements are lower than that of the refractory abundances, has been considered as a possible signature of a selfenrichment mechanism (Smith et al. 2001). This is based on the never proven assumption that stellar accretion only favors near star disk regions rich in refractory elements.

In Winter et al. (2007), we explored by means of numerical simulations stellar accretion caused by an inward forced planetary migration in a disk formed only by planetesimals that cause a metallic self-enrichment of the central star photosphere. At present, we attempt to identify this stellar accretion by determining the origin in the disk of these particles (planetesimals). 
In this way, we can separate the contributions of rocky material enriched differentially with refractory, intermediate, and volatile elements. In this work, we apply this methodology to three different systems, namely: CoRoT-2 (Alonso et al. 2008), 3 (Deleuil et al. 2008), and 4 (Aigrain et al. 2008). The main purpose of this work is to analyze the mentioned slopes of $[\mathrm{Fe} / \mathrm{H}] \mathrm{vs}$. $T_{\mathrm{C}}$ corresponding to CoRoT-2, CoRoT-3, CoRoT-4, and CoRoT5 and their interpretations. For cases 2 and 4, we obtained new high resolution spectra. Cases 3 and 5 are taken from the literature.

\section{Observations}

The high-resolution spectra of CoRoT-2 and CoRoT-4 analyzed in this work were obtained with the FEROS (Fiberfed Extended Range Optical Spectrograph) echelle spectrograph (Kaufer et al. 1999) of the $2.2 \mathrm{~m}$ ESO telescope at La Silla (Chile), on October 23th, 2008. The FEROS spectral resolving power is $R=48000$, corresponding to 2.2 pixel of $15 \mu \mathrm{m}$, and the wavelength coverage ranges from 3500 to $9200 \AA$. The nominal signal-to-noise ratio $(S / N)$ was evaluated by measuring the rms-flux fluctuation in selected continuum windows, and the typical values were $S / N$ 55-60 after $2 \times 3600 \mathrm{~s}$ of integration time. The spectra were reduced with the MIDAS pipeline reduction package consisting of the following standard steps: CCD bias correction, flatfielding, spectrum extraction, wavelength calibration, correction of barycentric velocity, and spectrum rectification.

\section{Abundance analysis of CoRoT-2 and CoRoT-4}

\subsection{Stellar parameters and iron abundances}

Determination of the basic, effective temperature $\left(T_{\text {eff }}\right)$, surface gravity $(\log g)$, microturbulence $\left(\xi_{\mathrm{t}}\right)$, metallicity $([\mathrm{Fe} / \mathrm{H}])$, and the abundance analysis were derived by means of the standard approach of the local thermodynamic equilibrium (LTE) using a revised version (2002) of the code MOOG (Sneden 1973) and a grid of Kurucz (1993) ATLAS9 atmospheres, which include overshooting.

The atmospheric parameters were obtained from the iron lines (Fe I and F II ) by iterating until the correlation coefficients between $\log \varepsilon(\mathrm{Fe} \mathrm{I})$ and lower excitation potential $(\chi l)$, and between $\log \varepsilon(\mathrm{Fe} \mathrm{I})$ and reduced equivalent width $\left(\log \left(W_{\lambda} / \lambda\right)\right.$ were zero, and the mean abundance given by Fe I and Fe II lines were similar. The iron lines taken from Lambert et al. (1996) and Santos et al. (2004) were carefully chosen by verifying that each line was not too strong, and checked for possible blending.

We adopted new $\log g f$ values for the iron lines. These values were computed from an inverted solar analysis using solar equivalent widths $(E W \mathrm{~s})$ measured from a solar spectrum taken with FEROS and a Kurucz grid model for the Sun (Kurucz $1993)$ having $\left(T_{\mathrm{eff}}, \log g, \xi_{\mathrm{t}}, \log \varepsilon(\mathrm{Fe})\right)=(5777 \mathrm{~K}, 4.44 \mathrm{dex}$, $\left.1.00 \mathrm{~km} \mathrm{~s}^{-1}, 7.47 \mathrm{dex}\right)$. Table 1 contains the linelist used, the adopted parameters, and also includes the $E W$ s measured in each star. Table 2 shows the atmospheric parameters derived by us and those found by other authors.

We determined equivalent widths using the "splot" task of IRAF $^{1}$. Abundances were computed using the ABFIND driver in MOOG.

\footnotetext{
${ }^{1}$ IRAF is distributed by the National Optical Astronomy Observatory, which is operated by the Association of Universities for Research in Astronomy, Inc., under cooperative agreement with the National Science Foundation.
}

Table 1. Atomic parameters of the iron lines.

\begin{tabular}{|c|c|c|c|c|c|}
\hline Species & $\lambda$ & $x_{l}$ & $\overline{l o g} g f$ & $\begin{array}{l}E W(\mathrm{~mA}) \\
\text { CoRoT-2 }\end{array}$ & $\begin{array}{c}E W(\mathrm{~mA}) \\
\text { CoRoT-4 }\end{array}$ \\
\hline \multirow{35}{*}{ Fe I } & 5044.22 & 2.85 & -2.043 & 88.0 & 66.7 \\
\hline & 5198.72 & 2.22 & -2.176 & & 94.5 \\
\hline & 5242.49 & 3.63 & -1.130 & 102.3 & 84.1 \\
\hline & 5322.05 & 2.28 & -2.900 & 77.2 & 56.3 \\
\hline & 5638.27 & 4.22 & -0.809 & 94.1 & 77.6 \\
\hline & 5731.77 & 4.26 & -1.124 & 73.0 & 53.2 \\
\hline & 5806.73 & 4.61 & -0.893 & 70.3 & 51.9 \\
\hline & 5852.22 & 4.55 & -1.185 & 52.2 & 36.2 \\
\hline & 5855.08 & 4.61 & -1.530 & 26.1 & \\
\hline & 5856.09 & 4.29 & -1.568 & 39.3 & \\
\hline & 6027.06 & 4.08 & -1.190 & 80.0 & 61.6 \\
\hline & 6056.01 & 4.73 & -0.500 & 92.2 & 73.2 \\
\hline & 6079.01 & 4.65 & -1.010 & & 39.7 \\
\hline & 6089.57 & 5.02 & -0.884 & 45.1 & 36.5 \\
\hline & 6151.62 & 2.18 & -3.300 & 70.6 & 40.2 \\
\hline & 6157.73 & 4.07 & -1.240 & 76.5 & 61.3 \\
\hline & 6159.38 & 4.61 & -1.860 & & \\
\hline & 6165.36 & 4.14 & -1.500 & 53.3 & 42.0 \\
\hline & 6180.21 & 2.73 & -2.635 & 71.7 & 54.0 \\
\hline & 6188.00 & 3.94 & -1.635 & 58.7 & 45.6 \\
\hline & 6200.32 & 2.61 & -2.400 & 98.0 & 65.9 \\
\hline & 6213.44 & 2.22 & -2.592 & 102.0 & 76.2 \\
\hline & 6226.74 & 3.88 & -2.070 & 33.6 & \\
\hline & 6229.24 & 2.84 & -2.880 & 45.5 & \\
\hline & 6265.14 & 2.18 & -2.574 & 111.0 & 77.0 \\
\hline & 6270.23 & 2.86 & -2.588 & 68.5 & 49.0 \\
\hline & 6380.75 & 4.19 & -1.320 & 66.9 & 51.9 \\
\hline & 6392.54 & 2.28 & -3.933 & 25.1 & \\
\hline & 6498.94 & 0.96 & -4.632 & 63.5 & 38.0 \\
\hline & 6627.55 & 4.55 & -1.480 & 32.1 & \\
\hline & 6703.57 & 2.76 & -3.027 & 43.1 & 31.3 \\
\hline & 6726.67 & 4.61 & -1.050 & 51.4 & \\
\hline & 6750.16 & 2.42 & -2.621 & 93.2 & 75.4 \\
\hline & 6752.71 & 4.64 & -1.230 & 47.0 & 36.7 \\
\hline & 6786.86 & 4.19 & -1.900 & 30.0 & 21.0 \\
\hline \multirow[t]{9}{*}{ Fe II } & 5234.63 & 3.22 & -2.238 & 96.4 & 100.8 \\
\hline & 5991.38 & 3.15 & -3.529 & & 47.9 \\
\hline & 6084.11 & 3.20 & -3.767 & & 33.3 \\
\hline & 6149.25 & 3.89 & -2.726 & 44.9 & 53.7 \\
\hline & 6247.56 & 3.89 & -2.342 & 64.5 & 72.2 \\
\hline & 6369.46 & 2.89 & -4.134 & 23.7 & \\
\hline & 6416.93 & 3.89 & -2.640 & 43.1 & 57.0 \\
\hline & 6432.69 & 2.89 & -3.564 & 44.9 & 52.9 \\
\hline & 7711.73 & 3.90 & -2.538 & 49.8 & \\
\hline
\end{tabular}

Notes. Column 1: element; Col. 2: wavelength (in $\AA$ ); Col. 3: excitation energy of the lower energy level in the transition (in eV); Col. 4: oscillator strengths based on an inverse solar analysis.; Col. 5: $E W$ for CoRoT-2; Col. 6: $E W$ for CoRoT-4.

\subsection{Stellar abundances}

We present here results for 15 elements (O, Li, Na, Mg, Al, Si, S, $\mathrm{Ca}, \mathrm{Sc}, \mathrm{Ti}, \mathrm{Cr}, \mathrm{Mn}, \mathrm{Ni}, \mathrm{Zn}$, and $\mathrm{Ba}$ ), plus Fe. The abundances of $\mathrm{Na}, \mathrm{Mg}, \mathrm{Al}, \mathrm{Si}, \mathrm{Ca}, \mathrm{Sc}, \mathrm{Ti}, \mathrm{Cr}, \mathrm{Mn}, \mathrm{Ni}, \mathrm{Zn}$, and Ba were derived from the analysis of $E W$ s for several unblended lines, measured by Gaussian fitting. To derive semi-empirical atomic $\log g f$ values for the lines of these elements, we used the $E W \mathrm{~s}$ measured in the solar spectrum and performed an inverted solar analysis, as that carried out for the iron element. The solar abundances of each element were taken from Anders \& Grevesse (1989). Table 3 summarizes the linelist, the atomic parameters adopted, and the $E W$ s measured in each star. For $\mathrm{Li}, \mathrm{O}$, and $\mathrm{S}$, we used a spectral synthesis procedure. 
Table 2. Stellar parameters of the CoRoT exoplanets parent stars.

\begin{tabular}{|c|c|c|c|c|c|c|c|}
\hline Star Name & $T_{\text {eff }}[\mathrm{K}]$ & $\log g$ & $\xi_{\mathrm{t}}\left[\mathrm{km} \mathrm{s}^{-1}\right]$ & {$[\mathrm{Fe} / \mathrm{H}]$} & {$[\mathrm{M} / \mathrm{H}]$} & $v \sin i\left[\mathrm{~km} \mathrm{~s}^{-1}\right]$ & References \\
\hline CoRoT-2 & $5696 \pm 70$ & $4.42 \pm 0.12$ & $1.71 \pm 0.10$ & $0.03 \pm 0.06$ & $0.03 \pm 0.06$ & $8.5 \pm 1$ & This work \\
\hline CoRoT-4 & $6115 \pm 70$ & $4.30 \pm 0.12$ & $1.37 \pm 0.10$ & $0.12 \pm 0.05$ & $0.10 \pm 0.05$ & $5.5 \pm 1$ & This work \\
\hline \multirow{2}{*}{ CoRoT-2 } & $5625 \pm 120$ & $4.30 \pm 0.20$ & - & - & $0.0 \pm 0.1$ & $11.85 \pm 0.45$ & Bouchy et al. (2008) \\
\hline & $5608 \pm 37$ & $4.71 \pm 0.20$ & $1.49 \pm 0.06$ & $0.07 \pm 0.04$ & $\begin{array}{c}0.0 .1 \\
-\end{array}$ & $\begin{array}{c}- \\
-1.00\end{array}$ & Ammler-von Eiff et al. (2009) \\
\hline CoRoT-4 & $6190 \pm 60$ & $4.41 \pm 0.05$ & $0.94 \pm 0.05$ & - & $0.05 \pm 0.07$ & $6.4 \pm 1$ & Moutou et al. (2008) \\
\hline CoRoT-3 & $6740 \pm 140$ & $4.22 \pm 0.07$ & - & $0.03 \pm 0.06$ & $-0.02 \pm 0.06$ & $17 \pm 1$ & Deleuil et al. (2008) \\
\hline CoRoT-5 & $6100 \pm 65$ & $4.189 \pm 0.03$ & $0.91 \pm 0.09$ & $-0.25 \pm 0.06$ & $-0.25 \pm 0.06$ & $1 \pm 1$ & Rauer et al. (2009) \\
\hline
\end{tabular}

Notes. Column 1: star name; Col. 2: effective temperature; Col. 3: surface gravity; Col. 4: microturbulence; Col. 5: metallicity; Col. 6: mean abundance (using element with more than three lines); Col. 7: projected rotational velocity; Col. 8: references.

It is important to study the highly volatile CNO elements because due to their low $T_{\mathrm{C}}$ values, they are indicators of the cool disk zone below $371 \mathrm{~K}$ (Lodders 2003). We took the condensation temperatures $\left(T_{\mathrm{C}}\right)$ from Lodders $(2003)$, covering a range between 70 and $1800 \mathrm{~K}$. An attempt to measure abundances for an important volatile element, such as $\mathrm{C}$, was unsuccessful. A reliable calculation of the $\mathrm{C}$ abundance requires a higher $S / N$ than used in this work. For instance, the $\mathrm{C}$ I lines in the region of $7115 \AA$ are hardly detectable and are severely blended. Similar problems are found for other $\mathrm{C}$ I lines, but we instead measured other volatile elements as $\mathrm{S}$ and $\mathrm{O}$ for both stars. The abundance ratios $[\mathrm{X} / \mathrm{H}]$ (and $[\mathrm{Fe} / \mathrm{H}]$ ) for each element, averaged over all useful lines, are presented in Table 4, along with the number of lines used in each case.

\subsubsection{Abundance uncertainties}

The internal errors in the adopted effective temperature and microturbulence can be determined from the uncertainty in the slope of both the Fe I abundance versus excitation potential, and the $\mathrm{Fe}$ I abundance versus reduced equivalent width relations. The standard deviation in $\log g$ was inferred by changing this parameter around the adopted solution until the and Fe II mean abundances differ by exactly one standard deviation of the mean value of the Fe I abundance. These quantities are given in Table 2.

Errors and uncertainties in the atmospheric parameters and determination for the continuum can affect abundance measurements in various ways. We tested the dependence of our results on atmospheric parameters obtained for CoRoT-2 and CoRoT-4.

Tables 5 and 6 show the variation in the abundance caused by $\Delta T_{\text {eff }}, \Delta \log g, \Delta \xi_{\mathrm{t}}$, and $\Delta W$. The sixth column indicates the combined rms uncertainty of the second to fifth columns. The last column indicates the observed abundance dispersion for elements whose abundances were derived using three or more lines.

We can observe that neutral elements are rather sensitive to temperature variations while single ionized elements are sensitive to the variations in $\log g$. For the elements whose abundance is based on stronger lines, such as barium, the error introduced by the microturbulence is significant. For the elements analyzed by means of spectrum synthesis the same technique was used, varying $T_{\text {eff }}, \log g$, and $\xi$, then independently computing the abundance changes introduced by the variation in the above atmospheric parameters.

The abundance uncertainties caused by the errors in the equivalent width measurements were computed from an expression provided by Cayrel de Strobel \& Spite (1988). The errors in the equivalent widths are determined, essentially, by the $S / N$ and the spectral resolution. In our case, having $R \approx 48000$ and a typical $S / N$ of 55-60, the expected uncertainties in the equivalent widths are about $3 \mathrm{~m} \AA$. These error estimates were applied to the measured $W_{\lambda}^{\prime}$ s and the corresponding changes in the element abundances are listed in Col. 5 of Tables 5 and 6.

The projected rotational velocities for both stars were determined by applying the synthetic spectra method to several single and clean lines. The results presented in Table 2 are the following: for CoRoT-2, we obtain $v \sin i=8.5 \pm 1 \mathrm{~km} \mathrm{~s}^{-1}$ and for CoRoT-4, we obtain $v \sin i=5.5 \pm 1 \mathrm{~km} \mathrm{~s}^{-1}$. These values can be compared with those obtained for the same stars by other authors. For CoRoT-4, Moutou et al. (2008) found $v \sin i=6.4 \pm 1.0 \mathrm{~km} \mathrm{~s}^{-1}$, which is similar to our value. The case of CoRoT-2 is different. Bouchy et al. (2008) determined three independent values for this parameter from different procedures: 1) $v \sin i=9.5 \pm 1.0 \mathrm{~km} \mathrm{~s}^{-1}$ from SOPHIE cross-correlation functions (CCFs); 2) $v \sin i=10.7 \pm 0.5 \mathrm{~km} \mathrm{~s}^{-1}$ from HARPS CCFs; and 3) $v \sin i=11.85 \pm 0.5 \mathrm{~km} \mathrm{~s}^{-1}$ by modeling the radial velocity anomaly occurring during the transit caused by the RossiterMcLaughlin (RM) effect. The value obtained using SOPHIE are in closer agreement with our value since the difference is within the error bars.

Column 4 of Table 2 shows the values of the microturbulence calculated for each star. We found a small discrepancy between the $\xi_{\mathrm{t}}$ that we found for CoRoT-4 $\left(1.37 \mathrm{~km} \mathrm{~s}^{-1}\right)$ and that found by Moutou et al. (2008) $\left(0.94 \mathrm{~km} \mathrm{~s}^{-1}\right)$, although both works show an approximate solar value. This difference may be caused by the number of iron lines and the method used for determining this parameter. Moutou et al. (2008) used the automatic VWA method (Bruntt et al. 2008), where they adjusted the microturbulence using an iterative procedure requiring that there is no correlation between the abundance and strength of a number of weak sensitive Fe I lines with equivalent widths $<80 \mathrm{~m} \AA$.

However, Moutou et al. (2008) are focused on presenting the discovery of the CoRoT-4b planet, and do not present a detailed analysis of the abundances values of the elements. We note, nevertheless, that our stellar parameters values, even if they have larger errors, are not incompatible with those of Moutou et al. (2008) in terms of $T_{\text {eff }}$ and $\log g$.

\subsection{Lithium}

Of the two observed stars, only CoRoT- 2 exhibits a relatively strong lithium resonance line with a measured equivalent width of $124 \mathrm{~m} \AA$. The secondary Li I line at $6104 \AA$ appears to be present in the spectrum as a very small feature in the wing of a Fe I line, confirming indirectly the high lithium abundance of 
Table 3. Atomic parameters of the spectral lines used for each element.

\begin{tabular}{|c|c|c|c|c|c|}
\hline Species & $\lambda$ & $\chi_{l}$ & $\log g f$ & $\begin{array}{c}E W(\mathrm{~mA}) \\
\text { CoRoT-2 }\end{array}$ & $\begin{array}{c}E W(\mathrm{~mA} \\
\text { CoRoT-4 }\end{array}$ \\
\hline \multirow[t]{3}{*}{$\mathrm{NaI}$} & 5688.22 & 2.10 & -0.678 & 133.7 & 110.3 \\
\hline & 6154.23 & 2.10 & -1.602 & 43.0 & 29.6 \\
\hline & 6160.75 & 2.10 & -1.316 & 64.0 & 43.0 \\
\hline \multirow[t]{2}{*}{ Mg I } & 5711.09 & 4.48 & -1.706 & 120.0 & 96.0 \\
\hline & 6318.72 & 5.21 & -1.996 & & 36.0 \\
\hline \multirow[t]{2}{*}{$\mathrm{Al}$ I } & 7835.31 & 4.02 & -0.738 & 43.0 & 30.0 \\
\hline & 7836.13 & 4.02 & -0.569 & 55.0 & 50.0 \\
\hline \multirow[t]{5}{*}{ Si I } & 5665.56 & 4.92 & -1.980 & 50.1 & 33.0 \\
\hline & 5948.55 & 5.08 & -1.197 & & 81.8 \\
\hline & 6142.49 & 5.62 & -1.518 & 35.3 & 28.0 \\
\hline & 6145.02 & 5.62 & -1.420 & 41.0 & \\
\hline & 6721.84 & 5.86 & -1.152 & 52.8 & 48.0 \\
\hline \multirow[t]{8}{*}{$\mathrm{Ca} \mathbf{I}$} & 5581.97 & 2.52 & -0.728 & 122.8 & 92.0 \\
\hline & 5590.12 & 2.52 & -0.815 & & 82.0 \\
\hline & 5867.56 & 2.93 & -1.599 & 33.0 & \\
\hline & 6166.44 & 2.52 & -1.171 & 84.0 & 63.0 \\
\hline & 6169.05 & 2.52 & -0.812 & 113.6 & 90.0 \\
\hline & 6169.56 & 2.52 & -0.567 & 145.7 & 103.0 \\
\hline & 6455.60 & 2.52 & -1.420 & 72.1 & 45.0 \\
\hline & 6471.66 & 2.53 & -0.848 & 118.0 & 86.0 \\
\hline \multirow[t]{3}{*}{ Sc II } & 5526.82 & 1.77 & 0.111 & 85.0 & 83.0 \\
\hline & 6245.65 & 1.51 & -1.051 & 37.0 & 33.0 \\
\hline & 6604.60 & 1.36 & -1.146 & & 41.5 \\
\hline \multirow[t]{5}{*}{ Ti I } & 5219.70 & 0.02 & -2.268 & 46.4 & \\
\hline & 5299.99 & 1.05 & -1.425 & 29.4 & 16.0 \\
\hline & 5866.46 & 1.07 & -0.827 & 70.0 & 34.0 \\
\hline & 6126.22 & 1.07 & -1.398 & 32.7 & 12.0 \\
\hline & 6258.11 & 1.44 & -0.450 & 59.9 & \\
\hline \multirow{6}{*}{$\mathrm{Cr} \mathrm{I}$} & 5296.70 & 0.98 & -1.411 & 115.0 & 84.0 \\
\hline & 5300.75 & 0.98 & -2.149 & 70.0 & 51.0 \\
\hline & 5345.81 & 1.00 & -1.036 & & 106.5 \\
\hline & 5348.33 & 1.00 & -1.301 & & 88.0 \\
\hline & 5783.87 & 3.32 & -0.220 & 60.0 & 40.0 \\
\hline & 5787.93 & 3.32 & -0.178 & 55.0 & 43.0 \\
\hline \multirow[t]{4}{*}{ Mn I } & 4265.92 & 2.94 & -0.442 & & 52.6 \\
\hline & 4470.13 & 2.94 & -0.585 & & 43.0 \\
\hline & 5399.47 & 3.85 & -0.098 & 44.2 & \\
\hline & 5432.54 & 0.00 & -3.636 & 60.0 & 33.0 \\
\hline \multirow[t]{6}{*}{ Ni I } & 5084.11 & 3.68 & -0.169 & 106.0 & 86.8 \\
\hline & 6327.60 & 1.68 & -3.100 & 43.2 & 38.1 \\
\hline & 6643.64 & 1.68 & -2.020 & 111.0 & 92.3 \\
\hline & 6767.77 & 1.83 & -2.170 & 91.0 & 77.5 \\
\hline & 6772.32 & 3.66 & -0.959 & 54.8 & 49.4 \\
\hline & 7788.93 & 1.95 & -1.951 & 101.2 & 88.3 \\
\hline \multirow[t]{2}{*}{$\mathrm{ZnI}$} & 4722.16 & 4.03 & -0.390 & 68.0 & 70.0 \\
\hline & 4810.53 & 4.08 & -0.310 & 87.0 & 75.0 \\
\hline \multirow[t]{3}{*}{ Ba II } & 5853.69 & 0.60 & -0.848 & 90.5 & 75.0 \\
\hline & 6141.73 & 0.70 & 0.146 & 160.0 & 130.0 \\
\hline & 6496.90 & 0.60 & -0.222 & 136.0 & 109.0 \\
\hline
\end{tabular}

Notes. Column 1: element; Col. 2: wavelength (in A ); Col. 3: excitation energy of the lower energy level in the transition (in eV); Col. 4: oscillator strengths based on an inverse solar analysis.; Col. 5: $E W$ for CoRoT-2; Col. 6: $E W$ for CoRoT-4.

this star. The lithium abundances were derived from synthetic spectra matching the observed Li I $6708 \AA$ resonance doublet.

The wavelengths and oscillator strengths for the individual hyperfine and isotope components of the lithium lines were taken from Smith et al. (1998) and Hobbs et al. (1999). The $\mathrm{CN}$ lines in the vicinity of the Li I doublet were included in the linelist. The solar ${ }^{6} \mathrm{Li} /{ }^{7} \mathrm{Li}$ isotopic ratio $\left({ }^{6} \mathrm{Li} /{ }^{7} \mathrm{Li}=0.081\right)$ (Anders \& Grevesse 1989) was adopted in the synthetic spectrum calculations.
Table 4. Abundances of CoRoT-2 and CoRoT-4.

\begin{tabular}{lcccc}
\hline \hline Species & $\begin{array}{c}\text { CoRoT-2 } \\
{[\mathrm{X} / \mathrm{H}]}\end{array}$ & $\begin{array}{c}\text { \# of } \\
\text { lines }\end{array}$ & $\begin{array}{c}\text { CoRoT-4 } \\
{[\mathrm{X} / \mathrm{H}]}\end{array}$ & $\begin{array}{c}\text { \# of } \\
\text { lines }\end{array}$ \\
\hline $\mathrm{O}$ I & $-0.13 \pm 0.10$ & 3 & $-0.03 \pm 0.10$ & 3 \\
$\mathrm{Na}$ I & $0.02 \pm 0.03$ & 3 & $0.05 \pm 0.10$ & 3 \\
$\mathrm{Mg}$ I & 0.04 & 1 & 0.1 & 2 \\
$\mathrm{Al} \mathrm{I}$ & -0.05 & 2 & -0.02 & 2 \\
$\mathrm{Si}$ I & $0.04 \pm 0.05$ & 4 & $0.01 \pm 0.08$ & 4 \\
$\mathrm{~S} \mathrm{I}$ & $0.00 \pm 0.09$ & 1 & $0.15 \pm 0.08$ & 1 \\
$\mathrm{Ca}$ I & $0.1 \pm 0.05$ & 7 & $0.1 \pm 0.05$ & 7 \\
$\mathrm{Sc}$ II & -0.04 & 2 & $-0.03 \pm 0.05$ & 3 \\
$\mathrm{Ti}$ I & $0.09 \pm 0.09$ & 5 & $0.06 \pm 0.11$ & 3 \\
$\mathrm{Cr}$ I & $0.01 \pm 0.08$ & 4 & $0.1 \pm 0.04$ & 6 \\
$\mathrm{Mn}$ I & -0.06 & 2 & $0.02 \pm 0.03$ & 3 \\
Fe I & $0.03 \pm 0.05$ & 32 & $0.12 \pm 0.05$ & 27 \\
Fe II & $0.02 \pm 0.07$ & 7 & $0.12 \pm 0.05$ & 7 \\
Ni I & $-0.08 \pm 0.06$ & 6 & $0.17 \pm 0.04$ & 6 \\
$\mathrm{Zn} \mathrm{I}$ & -0.1 & 2 & $0.01-$ & 2 \\
$\mathrm{Ba}$ II & $0.19 \pm 0.04$ & 3 & $0.14 \pm 0.06$ & 3 \\
\hline
\end{tabular}

Table 5. Abundance uncertainties of CoRoT-2.

\begin{tabular}{lcccccc}
\hline \hline Species & $\Delta T_{\text {eff }}$ & $\Delta \log g$ & $\Delta \xi$ & $\Delta W_{\lambda}$ & $\left(\sum \sigma^{2}\right)^{1 / 2}$ & $\sigma_{\text {obs }}$ \\
& $+70 \mathrm{~K}$ & +0.12 & +0.10 & $3 \mathrm{m \AA}$ & & \\
\hline Fe I & 0.05 & -0.01 & -0.02 & 0.05 & 0.07 & 0.05 \\
Fe II & -0.02 & 0.05 & -0.02 & 0.06 & 0.08 & 0.07 \\
Na I & 0.04 & -0.02 & -0.01 & 0.03 & 0.05 & 0.03 \\
Mg I & 0.04 & -0.03 & -0.02 & 0.03 & 0.06 & - \\
Al I & 0.02 & -0.02 & -0.01 & 0.03 & 0.04 & - \\
Si I & 0.01 & 0.01 & 0.0 & 0.04 & 0.04 & 0.05 \\
Ca I & 0.05 & -0.02 & -0.02 & 0.04 & 0.07 & 0.05 \\
Sc II & 0.0 & 0.05 & -0.02 & 0.05 & 0.07 & - \\
Ti I & 0.08 & 0.0 & -0.01 & 0.05 & 0.09 & 0.09 \\
Cr I & 0.06 & -0.01 & -0.03 & 0.05 & 0.08 & 0.08 \\
Mn I & 0.07 & 0.0 & -0.01 & 0.05 & 0.09 & - \\
Ni I & 0.05 & 0.0 & -0.03 & 0.05 & 0.08 & 0.06 \\
Zn I & 0.0 & 0.02 & -0.03 & 0.05 & 0.06 & - \\
Ba II & 0.02 & 0.03 & -0.05 & 0.05 & 0.08 & 0.04 \\
\hline Li I & 0.06 & 0.00 & 0.01 & - & 0.06 & 0.05 \\
O I & -0.03 & 0.07 & 0.00 & - & 0.08 & 0.10 \\
S I & -0.05 & 0.05 & 0.00 & - & 0.07 & 0.09 \\
\hline
\end{tabular}

Notes. The second column indicates the variation in the abundance caused by the variation in $T_{\text {eff }}$. The other columns refer, respectively, to the variations caused by to $\log g, \xi_{\mathrm{t}}$, and $W_{\lambda}$. The sixth column indicates the combined rms uncertainty of the second to fifth columns. The last column indicates the observed abundance dispersion for those elements whose abundances were derived using more than two lines.

For CoRoT-2, we obtained $\log \varepsilon(\mathrm{Li})=2.70 \pm 0.05$. The observed and synthetic spectra of CoRoT-2 in the region of Li I line are shown in Fig. 1. For the secondary Li I line, we only obtained the upper limit to the $\mathrm{Li}$ abundance of $\log \varepsilon(\mathrm{Li})<2.8$, which agrees with that obtained for the resonance line. The high $\mathrm{Li}$ abundance determined for CoRoT-2 of effective temperature $T_{\text {eff }}=5696 \mathrm{~K}$ and both its chromospheric activity and relatively high rotation velocity $v \sin i=8.5 \mathrm{~km} \mathrm{~s}^{-1}$ imply a young age of this star.

The star with the higher effective temperature $T_{\text {eff }}=$ $6115 \mathrm{~K}$, CoRoT-4, exhibits a relatively weak Li I resonance line. Analyzing the relatively noisy spectrum of this star in Fig. 2, we were unable to obtain a reliable value of the $\mathrm{Li}$ abundance. For CoRoT-4, we estimate only an upper limit to the lithium abundance on the order of $\log \varepsilon(\mathrm{Li})<2.2$. This apparently high $\mathrm{Li}$ abundance of CoRoT-4 is, however, consistent with the Li 
Table 6. Abundance uncertainties of CoRoT-4.

\begin{tabular}{lcccccc}
\hline \hline Species & $\Delta T_{\text {eff }}$ & $\Delta \log g$ & $\Delta \xi$ & $\Delta W_{\lambda}$ & $\left(\sum \sigma^{2}\right)^{1 / 2}$ & $\sigma_{\text {obs }}$ \\
& $+70 \mathrm{~K}$ & +0.12 & +0.1 & $3 \mathrm{~m} \AA$ & & \\
\hline $\mathrm{Fe} \mathrm{I}$ & 0.05 & -0.01 & -0.03 & 0.05 & 0.08 & 0.05 \\
$\mathrm{Fe}$ II & -0.01 & 0.04 & -0.04 & 0.05 & 0.08 & 0.05 \\
$\mathrm{Na}$ I & 0.04 & -0.01 & 0.0 & 0.05 & 0.06 & 0.10 \\
$\mathrm{Mg}$ I & 0.04 & -0.01 & -0.01 & 0.04 & 0.06 & - \\
$\mathrm{Al}$ I & 0.03 & -0.01 & 0.0 & 0.05 & 0.06 & - \\
$\mathrm{Si} \mathrm{I}$ & 0.03 & 0.0 & -0.01 & 0.05 & 0.06 & 0.08 \\
$\mathrm{Ca}$ I & 0.04 & -0.02 & -0.03 & 0.04 & 0.07 & 0.05 \\
$\mathrm{Sc}$ II & 0.0 & 0.04 & -0.03 & 0.05 & 0.07 & 0.05 \\
$\mathrm{Ti}$ I & 0.06 & -0.01 & -0.01 & 0.08 & 0.10 & 0.11 \\
$\mathrm{Cr}$ I & 0.06 & -0.01 & -0.04 & 0.06 & 0.09 & 0.04 \\
$\mathrm{Mn}$ I & 0.06 & 0.0 & -0.01 & 0.06 & 0.09 & 0.03 \\
$\mathrm{Ni} \mathrm{I}$ & 0.06 & -0.01 & -0.04 & 0.05 & 0.09 & 0.04 \\
$\mathrm{Zn}$ I & 0.03 & 0.0 & -0.05 & 0.06 & 0.08 & - \\
$\mathrm{Ba}$ II & 0.02 & 0.02 & -0.08 & 0.05 & 0.10 & 0.06 \\
\hline Li I & 0.05 & 0.00 & 0.00 & - & 0.05 & - \\
O I & 0.12 & 0.07 & 0.08 & - & 0.16 & 0.10 \\
$\mathrm{~S}$ I & -0.03 & 0.05 & 0.00 & - & 0.06 & 0.08 \\
\hline
\end{tabular}

Notes. The second column indicates the variation of the abundance caused by the variation in $T_{\text {eff }}$. The other columns refer, respectively, to the variations caused by $\log g, \xi_{\mathrm{t}}$, and $W_{\lambda}$. The sixth column indicates the combined rms uncertainty of the second to fifth columns. The last column indicates the observed abundance dispersion for those elements whose abundances were derived using more than two lines.

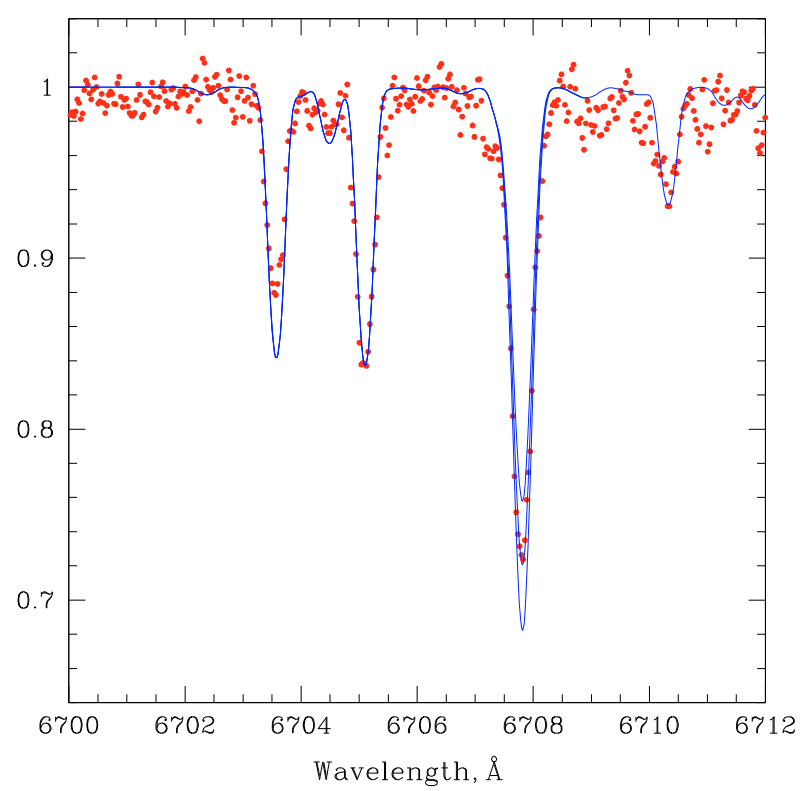

Fig. 1. CoRoT-2: observed (dotted red line) and synthetic (solid blue lines) spectra in the region of the Li I $6708 \AA$ line. Synthetic spectra were used to calculated the lithium abundances (from top to bottom): $\log \varepsilon(\mathrm{Li})=2.6,2.7$, and 2.8. The best-fit function to the observations is achieved for lithium abundance $\log \varepsilon(\mathrm{Li})=2.7 \pm 0.05$. We also show three iron absorption lines.

abundance of the main-sequence field and cluster stars with effective temperatures of $5900<T_{\text {eff }}<6300 \mathrm{~K}$ (the so-called Li-plateau region according to Boesgaard et al. 2004).

\subsection{Oxygen}

To determine of oxygen abundance, we used the O I triplet lines at 7771,7774 , and $7775 \AA$. The atomic data were taken from

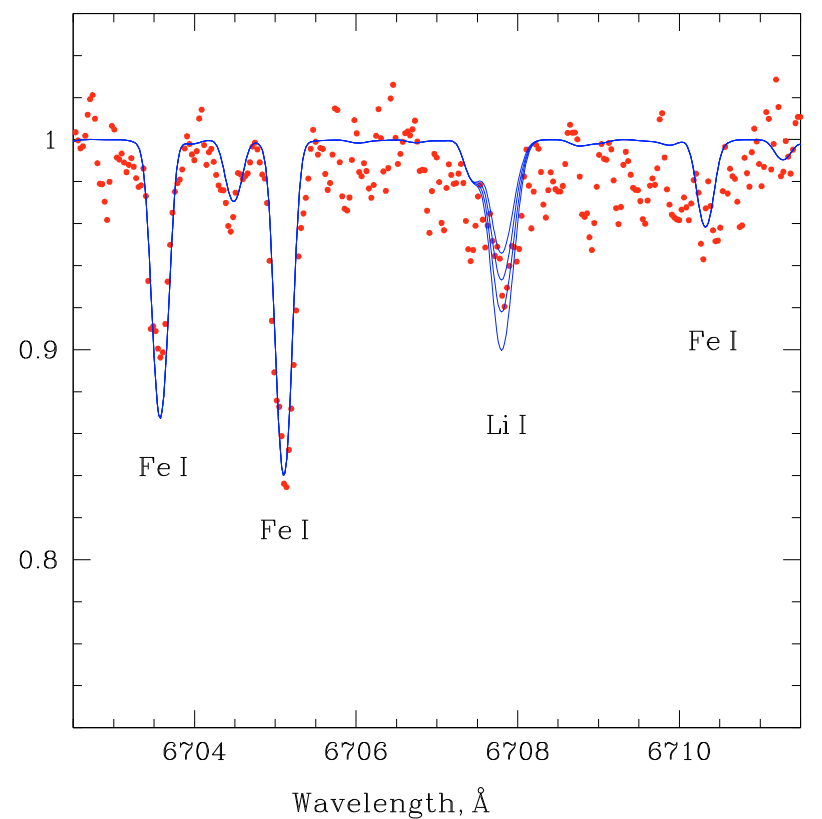

Fig. 2. CoRoT-4: observed (dotted red line) and synthetic (solid blue lines) spectra in the region of the Li I $6708 \AA$ line. Synthetic spectra were used to calculated the lithium abundances (from top to bottom): $\log \varepsilon(\mathrm{Li})=2.1,2.2,2.3$ and 2.4. It is difficult to determine a value for the abundance because of the noisy spectrum, and we only estimated an upper limit of $\log \varepsilon(\mathrm{Li})=2.2$. We also show three iron absorption lines.

the VALD database (Piskunov et al. 1995; Kupka et al. 1999). The value of the oscillation strength of the main component was modified to fit the solar abundance of $\log \varepsilon(\mathrm{O})=8.93$ (Anders \& Grevesse 1989).

The oxygen triplet lines are known to be affected by NLTE effects, which lead to an overestimation of the oxygen abundances. Even if the forbidden [O I] line at $6300.304 \AA$ is assumed to be free from NLTE effects, this line is blended with a telluric [O I] emission line in our spectra and cannot be used for the oxygen abundance determinations. To account for NLTE effects, we used the theoretical work of Takeda (2003), who calculated the values of the NLTE corrections for each line of the O I infrared triplet for a grid of different atmospheric parameters and equivalent widths of the oxygen lines.

For the cooler star of our sample, CoRoT-2, the NLTE correction is about 0.1 dex, whereas for the hotter star, CoRoT-4, NLTE effects are greater, about 0.2 dex. By applying the NTLE corrections, we derived the oxygen abundances $\log \varepsilon(\mathrm{O})=8.80$ and 8.90 for CoRoT-2 and CoRoT-4, respectively.

For CoRoT-2, not only a strong resonance Li I line but also emission in the Ca II lines is a strong indication of its youth and surface activity (see Fig. 3). In the case of CoRoT-4, emission is absent (Fig. 3). This age-related activity is proposed to affect the oxygen abundances derived from the oxygen triplet lines (Shen et al. 2007) and may also affect the precision of the oxygen abundance determination for CoRoT-2 star.

\subsection{Sulfur}

Sulfur is a very important volatile element, being one of the commonly and observed elements, the only reference element in an extended interval of $T_{\mathrm{C}}$ values between $704 \mathrm{~K}$ and $371 \mathrm{~K}$ (Lodders 2003). To obtain the $\mathrm{S}$ abundances in both stars, we selected the line of S I at $6757 \AA$. This line consists of three fine 

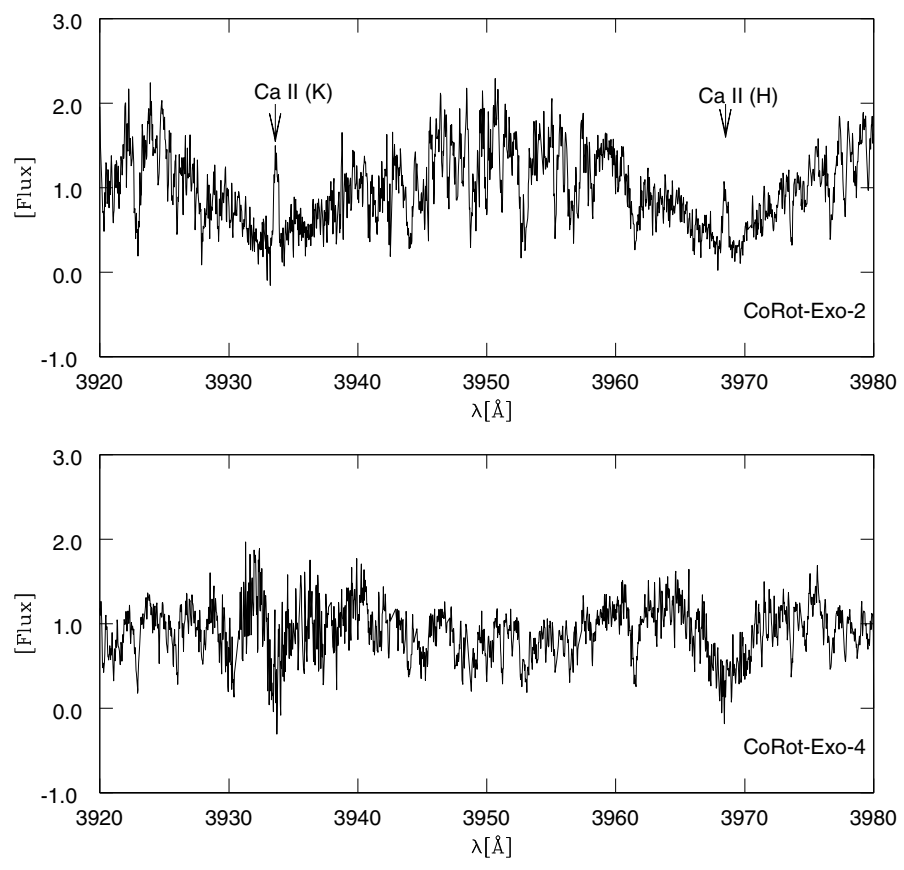

Fig. 3. Calcium II K and $\mathrm{H}$ lines for stars CoRoT-2 and CoRoT-4. The central emission features in the $\mathrm{Ca}$ lines correspond to the younger and chromospheric active CoRoT-2 star.

structure components and has to be studied using the synthetic spectrum method. We used the $\log g f$ data of Wiese et al. (1969), which were provided by VALD.

The value of the oscillation strength of the main component $(-0.310)$ was modified to -0.340 to fit the solar abundance of $\log \varepsilon(S)=7.21$ (Anders \& Grevesse 1989). The sulfur abundances that we derived are $\log \varepsilon(\mathrm{S})=7.21 \pm 0.09$ and $\log \varepsilon(\mathrm{S})=7.36 \pm 0.08$ for CoRoT-2 and CoRoT-4, respectively.

\section{Numerical simulations: analysis and methodology}

\subsection{The planetesimal disk}

A protoplanetary disk contains a mixture of gas and dust grains. These dust grains collide and build up larger and larger bodies, and small solid bodies known as planetesimals are presumably formed. Protoplanets grow within protoplanetary disks via pairwise accretion of planetesimals. When the mass of a protoplanet is of the order of 10-20 terrestrial masses, it can gravitationally capture gas-rich disk, thus producing Jovian-type planets. Protoplanetary disk is expected to lose its gas component in the first $10 \mathrm{Myr}$, leaving a disk formed essentially of dust and planetesimals.

For our purposes, we assumed that large planets observed close to their parent stars actually formed at larger distances but migrated inward due to energy loss and excess energy used to disperse the planetesimals (Murray et al. 1998). A detailed description of the planet migration used in this work is presented in Winter et al. (2007). It consist of a forced inward migration of a planet supposedly interacting with a planetesimal disk. Several possibilities were considered in terms of the planet eccentricities, migration rates, and mass. A more general result from Winter et al. (2007) is that 2:1 mean motion resonance is the main mechanism for driving planetesimals to the surface of the star. This mechanism operates for slow migration rates $\left(\tau=10^{5}-10^{6} \mathrm{yr}\right)$ and low planet eccentricities.
Table 7. Most important and more abundant rock-forming elements (from Lodders 2003).

\begin{tabular}{lccc}
\hline \hline Type of element & Element & $T_{\mathrm{C}}\left({ }^{\circ} \mathrm{K}\right)$ & $a(\mathrm{AU})$ \\
\hline Refractory & $\mathrm{Ca}, \mathrm{Al}, \mathrm{Ti}$ & $1650-1360$ & $0.03-0.04$ \\
Common & $\mathrm{Fe}, \mathrm{Si}, \mathrm{Mg}$ & $1360-1290$ & $0.04-0.046$ \\
Moderately Volatile & $\mathrm{P}$ & $1290-704$ & $0.046-0.15$ \\
Volatile & $\mathrm{S}$ & $704-371$ & $0.15-0.48$ \\
Highly Volatile & $\mathrm{H}, \mathrm{C}, \mathrm{N}, \mathrm{O}+$ & $<371$ & $0.48-<5$ \\
& $\mathrm{He}, \mathrm{Ne}, \mathrm{Ar}$ & & \\
\hline
\end{tabular}

To construct a rocky disk model valid for ages equal to or older than $10 \mathrm{Myr}$, several considerations must be taken into account. This disk containing a planetesimal distribution can be modeled using, for instance,knowledge obtained about a solar primeval nebula. Based on Lodders (2003), in Table 7 we present the distribution of the most important and more abundant rockforming elements. We indicate the type of elements (from highly refractory up to highly volatile), their proper elements, their interval of condensation temperature, and finally, their semi-major axis distribution (in AU) with respect to a central solar type star whose radial thermal distribution is valid for an age older than 10 Myr.

For simplicity, we assume that in a certain epoch of the disk evolution, most probably in the range 20-30 Myr (see Sect. 5), the disk is formed by a sea of planetesimals defining three representative zones: a refractory rich planetesimals zone $R$ between $\sim 0.03 \mathrm{AU}$ and $\sim 0.1 \mathrm{AU}$ (between $1780 \mathrm{~K}$ and $1360 \mathrm{~K}$ ), an intermediate zone I for particles between $\sim 0.1 \mathrm{AU}$ and $1.56 \mathrm{AU}$ (1360 K to $200 \mathrm{~K})$, and a zone $\mathrm{V}$ of volatiles between 1.56 AU and $4.5 \mathrm{AU}(<200 \mathrm{~K})$.

In reality, the situation must be far more complex. Because nebular turbulence and dust migration probably occur during the earlier nebular stages, a radial mixture is expected. If zone $R$ contains the pure refractory elements, zone $I$ must contain a true mixture of elements. An example of this mixture in zone $I$, is the presence of Triolite $(\mathrm{FeS})$ at $0.15 \mathrm{AU}$ with a $T_{\mathrm{C}}=700 \mathrm{~K}$ (Lodders 2003). At this distance, an important condensation of pure $\mathrm{Fe}$ grains and a mild condensation of pure $\mathrm{C}$ may also occur (Wehrstedt \& Gail 2002). The zone $I$ also contains the internal end of the migration condensation front of $\mathrm{H}_{2} \mathrm{O}$ particles at $\sim 0.8 \mathrm{AU}$ coming from the external zones of the disk (Davis 2005). In summary, we can consider that zone $I$ contains planetesimals composed of a mixture of refractory type grains and volatile ices. The zone $\mathrm{V}$ is characterized by the abundant highly volatile CNO elements for which the highest $T_{\mathrm{C}}$ is only $180 \mathrm{~K}$ for oxygen.

\subsection{The planet migration}

We numerically integrated the CoRoT-2, CoRoT-3, and CoRoT4 systems within the circular restricted three-body problem of star-planet-planetesimals. The orbital and physical parameters for these stars are listed in Table 8. Each parameter is listed along with its formal uncertainty. We assumed that the planet may have been formed at a distance between 2 and $5 \mathrm{AU}$, where they are supposed to form under conditions where condensible materials exist. Thus, the planet is set initially with semi-major axes $a_{P_{\mathrm{i}}}=2,3,4$, and $5 \mathrm{AU}$ and eccentricity $e_{P}=0.0$. The planet is forced to migrate inward to its current position, $a_{P_{\mathrm{f}}}$, with constant speed in a time-scale $\tau$. We assumed that the planetesimal disk is composed of 1000 test massless particles moving around the star on initially randomly distributed circular orbits. The disk 
Table 8. Orbital and physical parameters of the stellar systems.

\begin{tabular}{lccccc}
\hline \hline Orbital parameter & $M_{\text {star }}\left(M_{\odot}\right)$ & $M_{\text {planet }}\left(M_{\text {Jupiter }}\right)$ & Semi-major axis (AU) & Eccentricity & References \\
\hline Corot-2b & $0.97 \pm 0.06$ & $3.31 \pm 0.016$ & $0.0281 \pm 0.0009$ & 0.0 (fixed) & Bouchy et al. (2008) \\
Corot-3b & $1.37 \pm 0.09$ & $21.66 \pm 1.0$ & $0.057 \pm 0.003$ & 0.0 (fixed) & Deleuil et al. (2008) \\
Corot-4b & $1.16_{-0.02}^{+0.03}$ & $0.72 \pm 0.08$ & $0.09 \pm 0.001$ & $0.0_{-0.0}^{+0.1}$ & Moutou et al. (2008) \\
\hline
\end{tabular}

Table 9. Total mass of planetesimals for the planet migration up to its current position in the stellar system.

\begin{tabular}{lccc}
\hline \hline Disk mass $\left(M_{D}\right)$ & Corot-2b & Corot-3b & Corot-4b \\
\hline$a_{P_{\mathrm{i}}}(\mathrm{AU})$ & $2-5$ & $2-5$ & $2-5$ \\
$M_{\text {Disk }}\left(M_{\odot}\right)$ & $0.013-0.016$ & $0.075-0.093$ & $0.002-0.003$ \\
\hline
\end{tabular}

is interior to the orbital radius of the planet. The newly formed planet is surrounded by an annular gap in the planetesimal disk (Wisdom 1980; Lissauer 1993). We assumed here an annular gap of 0.5 AU relative to the outer disc edge. Therefore, the outer radius is, in all cases, $0.5 \mathrm{AU}$ smaller than the initial position of the planet. Thus the disk outer radius $\left(a_{D_{f}}\right)$ is located at 1.5, 2.5, 3.5, and 4.5 AU, according to the planet initial semi-major axis. The inner radius $\left(a_{D_{\mathrm{i}}}\right)$ of the disk is located at $0.03 \mathrm{AU}$.

We are interested in studying the relationship between the metallicity of these CoRoT systems and the migration resulting from interaction with planetesimals. Since a part of the mass of planetesimals will be accreted by the star during migration, we need to estimate for these CoRoT systems the mass of the disk contained inside the orbit of each planet. Considering the initial semi-major axes of the planets, we calculated the orders of the total mass $M_{D}$ of the planetesimal disk required for the planet to migrate to its current position (Adams \& Laughlin 2003). These data are presented in Table 9 . We assumed that about $1 \%$ of the disk is in the form of solid bodies called planetesimals (Davis 2005). We note that the order of the total mass of the planetesimal disk increases with planetary mass and larger $a_{P_{\mathrm{i}}}$. Using these values, we now estimate the percentage of the element types that fall on the star during the planet migration. The numerical code SWIFT (Levison \& Duncan 1994) was used. The integrator is based on the MVS method developed by Wisdom \& Holman (1991).

In general, the numerical simulations were performed for a timescale of $10^{5}$ years. The integration for each particle was interrupted whenever one of the following situations occurred:

(i) collision between the planetesimal and the planet, i.e., when the planetesimal becomes closer than 1.2 planetary radii from the planet;

(ii) collision between the planetesimal and the star, when the planetesimal becomes closer than 2.5 stellar radii from the star;

(iii) ejection from the system, when the planetesimal reaches more than $50 \mathrm{AU}$ from the star.

\subsection{Results}

The results of the numerical simulations are presented in Fig. 4. In that figure, we show the dependence of the percentage of planetesimals that fall on the star in terms of the value of $a_{P_{\mathrm{i}}}$. The main significance of the results are the statistics in terms of percentage of collisions with the star of planetesimals characterized by $R, I$, and $V$ as a function $a_{P_{\mathrm{i}}}$. The values of the percentages were obtained by measuring the number of planetesimals of the $R, I$, and $V$ zones that collided with the star. Thus the percentage of collisions of planetesimals with the star gives an estimate of the mass of each type of material that the star will accrete due to the migrating planet. We note that while this percentage provide reasonable estimates of the accreted mass, the assumption of there being a constant migration is not strictly correct since these collisions will tend to decrease as the planet migrates inwards.

We can also note from Fig. 4 that the percentage of planetesimals in the volatile zone that fall on the star is increases with increasing value of $a_{P_{\mathrm{i}}}$. This is due to the disk model considered. As mentioned in the previous section, the circumstellar disk is assumed to contain highly volatile particles between 1.56 and 4.5 AU. They are distributed in that region for values of the initial position of the planet beyond $2 \mathrm{AU}$. Thus, there are fewer particles in the volatile zone when the planet has an initial position $2 \mathrm{AU}$. In this case, all the particles are practically initially distributed in refractory and intermediate zones. Since the size of the refractory zone is much smaller (0.03-0.1 AU) than the size of the intermediate zone (0.1-1.56 AU), there is a much larger quantity of particles in the intermediate zone. A more significant number of particles is then expected to fall on the star from the intermediate zone. Therefore, our results show that for these CoRoT systems, particles from the intermediate zone dominate the collision process onto the star. In total about $79 \%$ of planetesimals from the intermediate zone were found to be falling onto the star. In the CoRoT- 2 and CoRoT-3 cases, all material ( $\sim 5 \%)$ of planetesimals from the refractory zone fell onto the star.

For all the migration cases of the planet of the CoRoT- 4 system, we note that planetesimals from the refractory zone did not fall onto the star. This occurred because the planet migration halts before the 2:1 resonance reaches the refractory zone.

When planets migrated from the initial position at $3 \mathrm{AU}$, about $3 \%$ of the planetesimals from the refractory zone fell onto CoRoT-2 and CoRoT-3 stars. Planetesimals from the volatile and intermediate zones fell on the stars. The results show that about $13 \%$ belongs to the volatile zone, while $59 \%$ belongs to the intermediate zone. For the initial positions of the planet at 4 and $5 \mathrm{AU}$, our results do not show significant differences between the values of percentages of planetesimals from the refractory, intermediate, and volatile zones that fall on CoRoT-2 and CoRoT-3. In these two cases, the numerical estimates are very close. There is doubtless no dependence of these percentages values with the initial semi-major axis of the planet. In general, giant planets are, therefore, be expected to form at distances between 4 and 5 AU. We focused on the situation of an orbital radius at $5 \mathrm{AU}$, where giant planets form and gain most of their mass.

Motivated by the much smaller total mass of its planetesimal disk than the other systems (Table 9), the numerical simulations for the CoRoT-4 case were also performed for timescales of $10^{3}$ and $10^{4}$ years. For very rapid migrations, the planetesimals experience close approaches to the planet and a significant number of them are ejected from the system (Winter et al. 2007). The question that we wish to answer is: how much mass from the disk will the star accrete during these times? The results are shown in Fig. 5. In this case, we can note that $26 \%$ of planetesimals from 

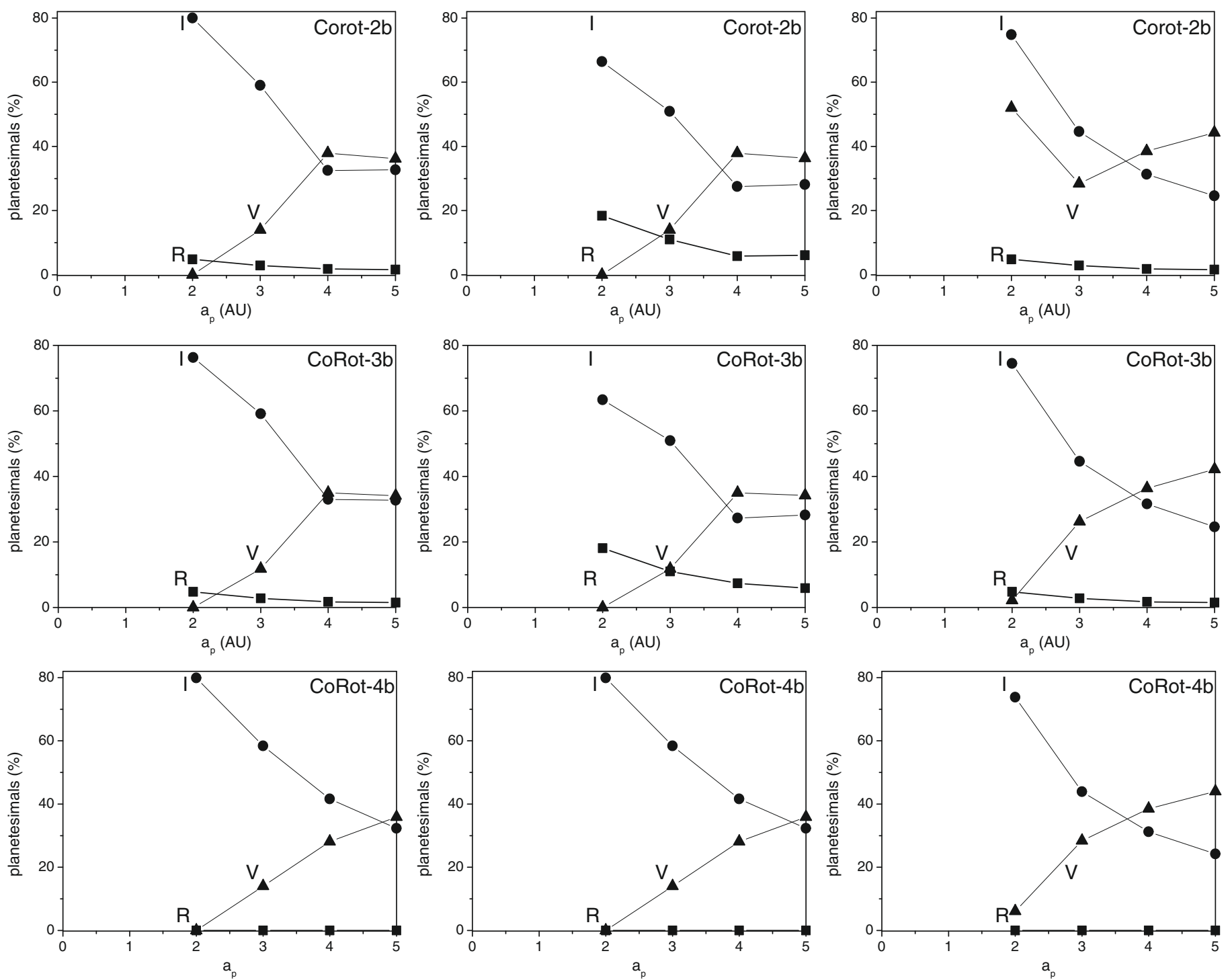

Fig. 4. These figures show the percentage of planetesimals that collided with the star of the CoRoT systems. Each curve gives the percentage of planetesimals of the zones refractory (square), intermediate (circle) and volatile (triangle). The values are given as a function of the initial semi-major axis of the planet, $a_{P_{\mathrm{i}}}$. At the top of each figure, we indicate the corresponding system. In the first column, the results are for the case of zones R between $0.03 \mathrm{AU}$ and $0.1 \mathrm{AU}$, I between $0.1 \mathrm{AU}$ and $1.56 \mathrm{AU}$, and $\mathrm{V}$ between $1.56 \mathrm{AU}$ and $4.5 \mathrm{AU}$. The second column is for the case of zones R between $0.03 \mathrm{AU}$ and $0.3 \mathrm{AU}$, I between $0.3 \mathrm{AU}$ and $1.56 \mathrm{AU}$, and $\mathrm{V}$ between $1.56 \mathrm{AU}$ and $4.5 \mathrm{AU}$. The third column is for the case of zones R between $0.03 \mathrm{AU}$ and $0.1 \mathrm{AU}$, I between $0.1 \mathrm{AU}$ and $1.2 \mathrm{AU}$, and $\mathrm{V}$ between $1.2 \mathrm{AU}$ and $4.5 \mathrm{AU}$.

the intermediate zone collided with star for $\tau=10^{4}$ years, while for $\tau=10^{3}$ years the value is about $3.9 \%$.

\subsection{Parameters dependence}

Until now, the results presented are concerning a given set of parameters model. In order to gain more insight into the numerical results from our disk simple model, it is useful to characterize and, when possible, quantify its dependence with respect to some of the parameters used. To investigate the sensitivity of our results, in this section we present a study about the influence of some of the adopted parameters on the amount of planetesimals that fall on the star.

\subsubsection{R, V and, I location zones}

A starting point for our discussion is provided by the distribution model of planetesimals in the disk, which is given by the location of the R, I, and V zones. This has important consequences. It implies that the percentage of the type of elements that fall on the star seems to be uniquely controlled by the location zones considered. As mentioned in Sect. 4.1, in reality a radial mixture of bodies of zones $\mathrm{R}$ and $\mathrm{V}$ must be contained in the whole disk. The intermediate zone is characterized by this mixture. Thus, we studied the sensitivity of our results to the location of the zones considered. It is therefore important to know how different locations of the R, I, and V zones affect the percentages of collisions of planetesimals with the star. To test the dependence of the results on the location zones, we relaxed the borders of the locations by considering 2 cases. In the first case, we moved the border between refractory and intermediate from $0.1 \mathrm{AU}$ to $0.3 \mathrm{AU}$. In the second case, we moved the border between intermediate and volatile from 1.56 AU to 1.2 AU.

Our results suggest that there are significantly change in the percentage values for the CoRot- 2 and CoRot- 3 systems (see second and third columns of Fig. 4). In these cases, it can be 

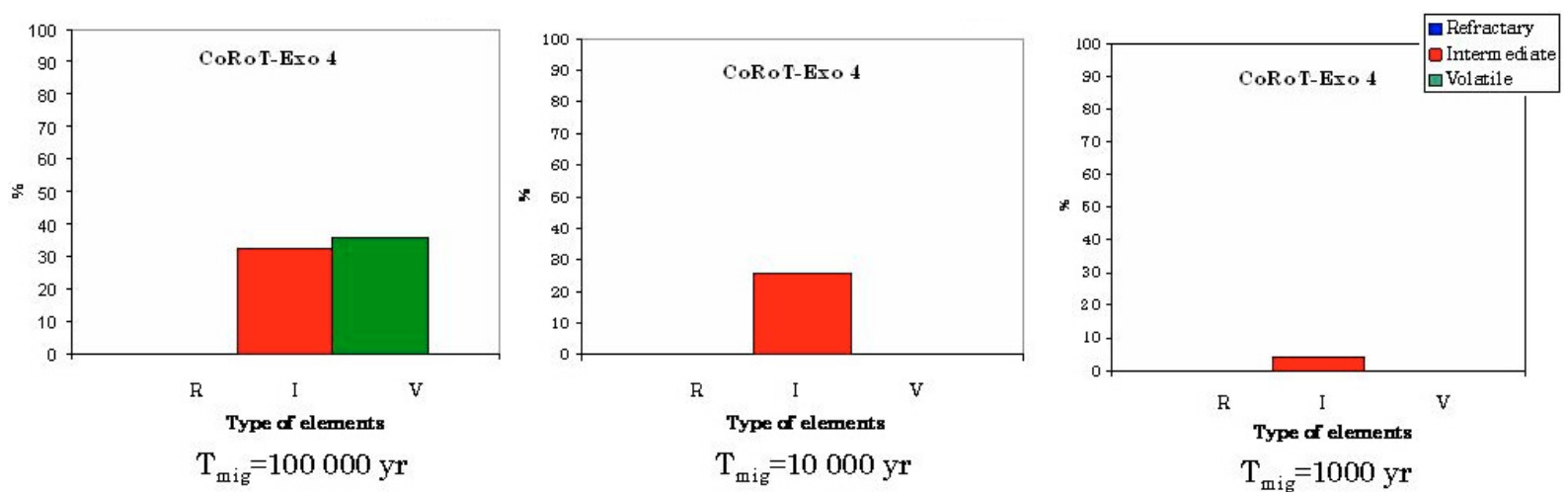

Fig. 5. Histogram of the percentage of planetesimals from the refractory (R), intermediate (I), and volatile (V) zones that fall on Corot-4 for the case of $a_{P_{\mathrm{i}}}=5 \mathrm{AU}$.

seen that a combination of higher and lower values of the initial distribution borders $\mathrm{R}$ and I (or I and V) results in an abrupt transition between refractory and intermediate elements (or intermediate and volatile elements) occurring at the positions that we defined as the borders. Thus, a reasonable fraction of particles near the borders change from "intermediate" to "refractory" elements (or from "intermediate" to "volatile" elements). In the first case, the percentage of refractory material that fall onto the star is increased by a fraction and the intermediate material that fall on the star is decreased by that same fraction. The same idea is valid for the second case, a fraction of intermediate material near the border is classified as volatile material. Then, the percentages of intermediate and volatile material are increased and decreased by the same amount, respectively.

For CoRot-4 system, there is only a change in the percentage values when the border between intermediate and volatile material is moved from 1.56 AU to 1.2 AU. There is no change in the percentage of refractory and volatile materials that fall onto the star when the border between refractory and intermediate zones is moved. This is because the mean-motion resonances play the main role in the orbital evolution of the planetesimals and their locations are unchanged by the shift of the borders. In this scenario, there is no change in the distribution of the initial orbital semi-major axis of the particles, but only a determination of the new location of the R, V, and I zones.

\subsubsection{Planet on eccentric orbit}

Another point for our discussion is provided by the planet's eccentricity $e_{P}$. We recall that the value of $e_{P}$ for any extrasolar planet can vary significantly due to uncertainties in the current observational methods of detection. As we can see in Table 9, the value of $e_{P}$ for CoRot-3b and CoRot-2b were assumed to be zero (Alonso et al. 2008; Deleuil et al. 2008). Thus, it is important to know whether different values of $e_{P}$ yield different evolutions. Winter et al. (2007) presented a numerical study about the influence of $e_{P}$ on capture of planetesimals in mean motion resonances during the planet migration. They showed that for $M_{\text {planet }}=M_{\text {Jupiter }}$, when $e_{P}=0$, the planetesimals are caught on $3: 2,5: 3$, or $2: 1$ resonances. The $2: 1$ resonance is responsible for the majority of impacts on the star. For $e_{P}=0.1$, the 5:2 and 3:1 are also important, but the $2: 1$ resonance is strong enough to dominate the orbital evolution of the planetesimal. In the case $e_{P}=0.2$, the $4: 1$ resonance is important, while the $3: 2$ and 5:3 resonances contribute very little to the evolution of the planetesimals. Other resonances also become important for the cases $e_{P}=0.3,0.4$, and 0.5 . Therefore, the orbital evolution of the disk of planetesimals is affected by several resonances but not dominated by any particular one. In these cases, the planetesimals with semi-major axis larger than $0.65 \mathrm{AU}$ are spread without being captured in resonance. Until now, we have assumed that the CoRot systems have $e_{P}=0$. A comparison of Fig. 4 with the results of Fig. 6 from Winter et al. (2007) for $e_{P}=0$ and $\tau=10^{5} \mathrm{yr}$ show that, in general, the percentage of planetesimals that fall on the star is between about of 70 and $80 \%$. Winter et al. (2007) demonstrated that this result is a direct consequence of the value of the migration speed.

We also studied the evolution of the system by considering the planet to be on an eccentric orbit. In Fig. 6, we present the results for $e_{P}=0.1,0.3$, and 0.5 , respectively. As expected for a circular orbit (see first column of Fig. 4) the percentage of collisions with the star decreases (from about $10 \%$ to $35 \%$ ) when the planet's eccentricity is increased. In general, we note that the percentage of intermediate and volatile materials accreted decrease. For the CoRot-3 system, the integrations stopped when the last planetesimal was removed from the system. The planet did not complete its migration at its current position. The same occurred for the planet from CoRot- 2 system when $e_{P}=0.5$. In both systems, the percentage values of refractory material do not differ from those for $e_{P}=0$. In the CoRot- 4 case, some planetesimals had survived when the integrations finished, but the planet arrived at its current position, $a_{D_{\mathrm{f}}}=0.09$ AU. In this case, we note an increase in the percentage of refractory material that fall on the star. Planetesimals in the refractory zone are captured in mean motion resonances and some of them fall onto the star.

A planet on an eccentric orbit can significantly increase the eccentricities of the planetesimals because of its gravitational perturbation force. When planetesimals reach very high eccentricities they are then removed from the system by collision or ejection. As shown in Winter et al. (2007), a fraction of planetesimals are spread without being captured in resonances. On the other hand, other planetesimals are captured in several resonances $(2: 1,3: 1,7: 2,5: 1,6: 1$, and 5:2). Planetesimals in higher resonances are not so well protected against close approaches with the planet and a fraction of them are ejected from the system.

\subsubsection{Warm planetesimals disk}

Here we consider a disk of planetesimals with eccentricities initially distributed between 0 and 0.1 . The results are presented in Fig. 7. We note that the results are similar to those of a disk of planetesimal initially in a circular orbit about a planet with an eccentricity of 0.1 (first column of Fig. 6). The differences between 

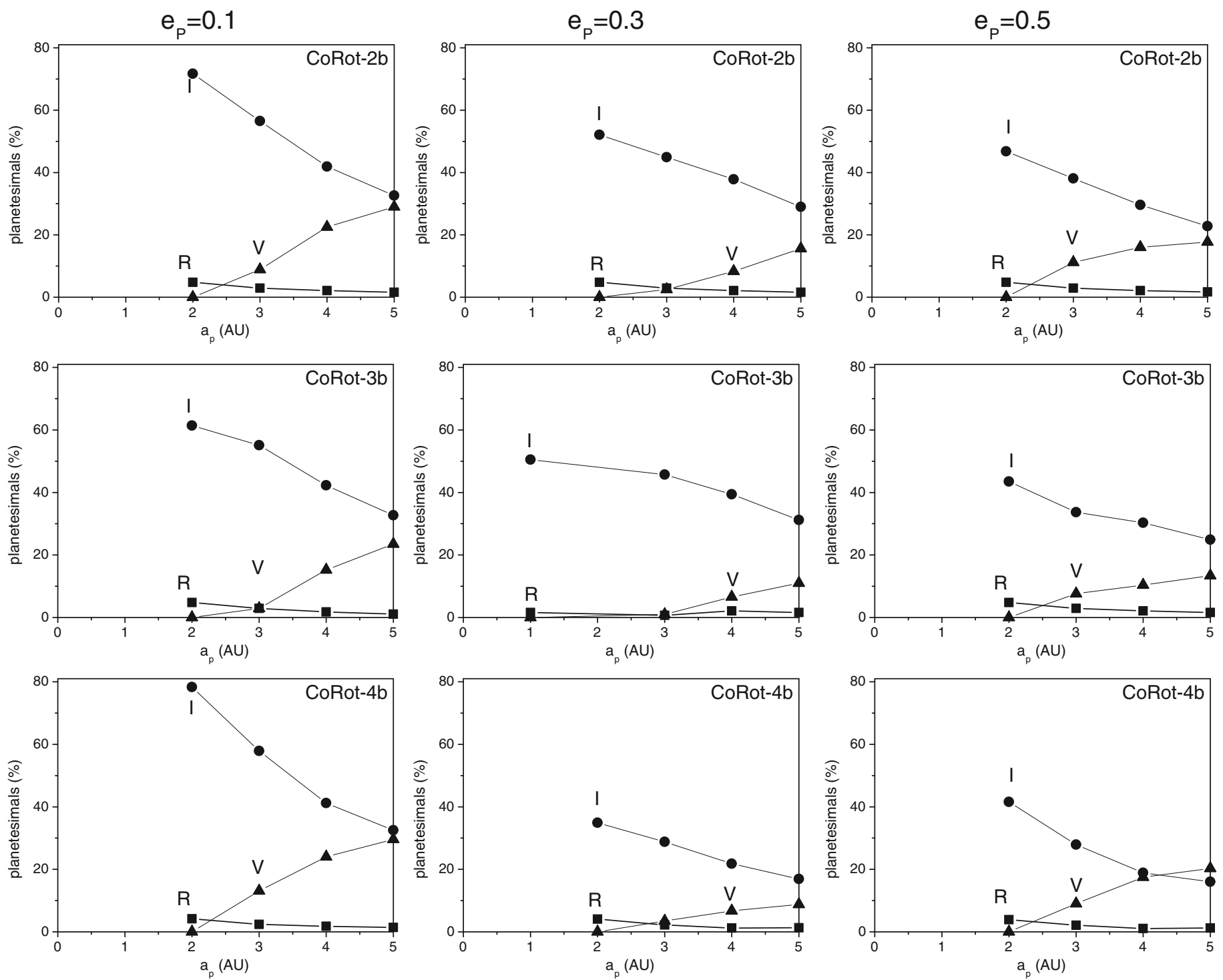

Fig. 6. Same as the first column of Fig. 4, but for planets initially at eccentric orbits. In the first, second and third columns the results are for $e_{P}=0.1,0.3$, and 0.5 cases, respectively.

these results and those from the first column of Fig. 4 are due to the amount of refractory material that fall on the star. Here, only the CoRot- 4 system has a percentage value of refractory material similar to those for $e_{P}=0.0$. In the present case, planetesimals in the refractory zone are ejected from the system. In the CoRot-2 and CoRot-3 systems, there is also a decrease in the amount of refractory material that falls onto the star, which was also ejected from the system. That is because the eccentricity (of the planetesimal or the planet) affects the capture in mean motion resonance between the planetesimal and the planet. We learned from Winter et al. (2007) that the 2:1 resonance is responsible for the majority of impacts onto the star. According to Quillen (2006), a body is captured into the $2: 1$ resonance when its initial eccentricity is below a certain limit given by $e_{\lim } \sim 1.5 \mu^{1 / 3}$. This is about $0.126,0.220$, and 0.371 for CoRoT-4, CoRoT-2, and CoRoT-3 systems, respectively.

\section{The link between migration and stellar abundances}

We now discuss some assumptions and physical conditions that are the basis of the link between the consequences of the type of planetary migration considered and the distributions of the elemental abundances relative to the condensation temperatures $T_{\mathrm{C}}$. To test this link, we present in Fig. 8 the abundances as a function of $T_{\mathrm{C}}$ for the CoRoT stars.

At a certain phase of the disk evolution, we assume that the disk is homogeneously filled with planetesimals between a minimal typical distance of $\sim 0.03 \mathrm{AU}$ and a maximal one of $\sim 4.5 \mathrm{AU}$. We also assume that a giant planet is formed at $\sim 5 \mathrm{AU}$, before migration. An age of the system (star + disk) of 20 to $30 \mathrm{Myr}$ is critical for the efficiency of this process (Winter et al. 2007). At this age, when the disk is already devoid of its gas component, a low mass central star will indeed reduce, for the first time, its external convective layer to its minimum configuration (see for instance Ford et al. 1999). In this situation, any metallic content of the planetesimals falling onto the star will probably remain in the photosphere of the star contributing to its metallic enrichment. This may also be true for ages older than $30 \mathrm{Myr}$, but not younger than $20 \mathrm{Myr}$ because any enrichment would then be drastically reduced due to the larger convective layer at these early epochs.

Clearly, the stellar enrichment depends on the quantity of infall material. If this mass were of the order of 35 Earth masses of 

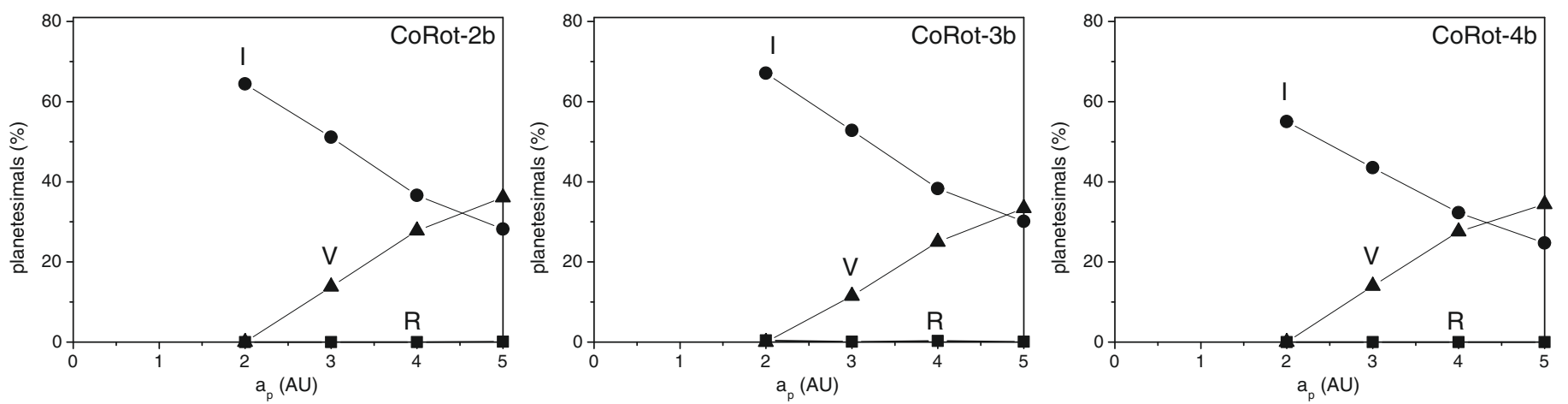

Fig. 7. Same as the first column of Fig. 4, but for planetesimals initially distributed at eccentricities between 0 and 0.1 .
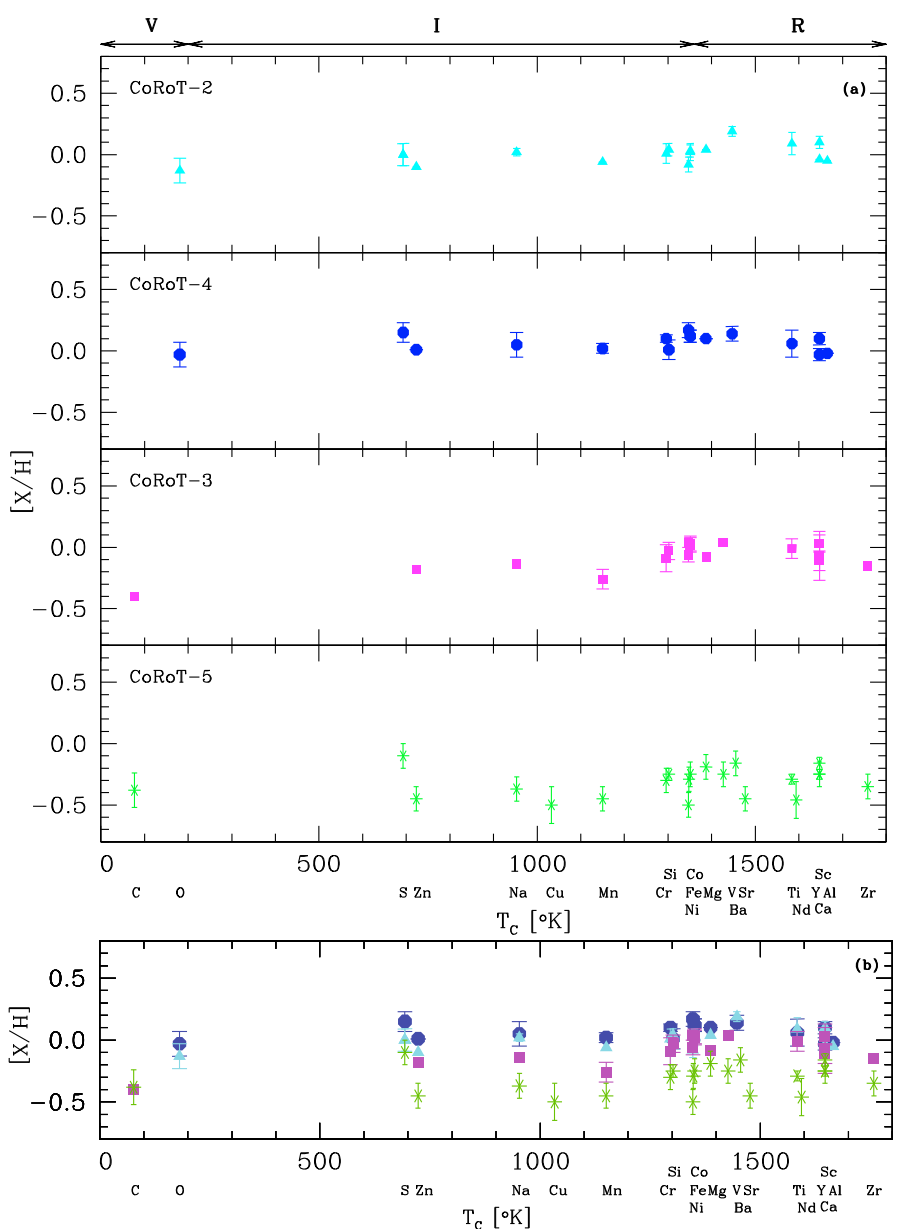

Fig. 8. a) Relative abundances distribution of elements for the CoRoT stars as a function of their condensation temperatures (separated). b) Superposed distributions: CoRoT-2 (cyan triangles), CoRoT-4 (blue circles), CoRoT-3 (magenta squares) and CoRoT-5 (green stars).

planetesimals consisting essentially of iron, this induced migration mechanism could furnish an iron enrichment of the order of 1.8 dex, as observed in stars hosting hot Jupiters (Winter et al. 2007). But, how would this affect the infall of a collection of rocky materials of varying richnesses of refractory, intermediate, and volatile elements? First, we must consider that in general, stars apparently without detected planets exhibit flat distributions (zero slope) of the element abundances with respect to $T_{\mathrm{C}}$ (Ecuvillon et al. 2006). Then, in principle, any differential stellar accretion will produce a positive slope of $[\mathrm{X} / \mathrm{H}]$ versus $T_{\mathrm{C}}$ if the volatile contribution is smaller than the refractory contribution. In contrast, negative slopes should appear if the volatile contribution is far more important.

Observationally speaking, also in the case of SWP, flat distributions are universally found (Ecuvillon et al. 2006). It is mainly for this reason that zero gradient distributions are, in the literature, synonymous with the absence of a self-enrichment mechanism. Nevertheless, Smith et al. (2001) found, using a collection of abundance data from the literature, that about six stars exhibit positive slopes. Other authors (Gonzalez 2006a,b; Sadakane et al. 2002; Takeda et al. 2001) found no dependence of the abundances on $T_{\mathrm{C}}$.

Applying our differential accretion mechanism methodology to three CoRoT systems (2, 3, and 4) considered in this paper, we found surprisingly that in all cases the volatile and intermediate contributions were very similar and much larger than the refractory ones (Fig. 4). Here, we present the results for a relatively slow planetary migration of $10^{5}$ years. As shown in Winter et al. (2007), for shorter migration times, the accretion mechanism is less efficient. We show this in Fig. 5 only for the case of CoRoT4 , which involves in the infall of intermediate material only.

At the top of Fig. 8, we have separated by arrows along the $T_{\mathrm{C}}$ scale, the three $\mathrm{V}, \mathrm{I}$, and $\mathrm{R}$ domains. In general, we can infer that similar $\mathrm{V}$ and I contributions represent a flat distribution of the elements in these two zones. In case of significant stellar accretion, the flat distribution will only be shifted upward as a whole. In the case of modest or null accretion, the original and flat distribution will be maintained. This is in fact, the case of CoRoT systems 2 and 3, where migration, at least in these conditions is not realized (see Sect. 6). Only in the case of CoRoT4 may differentiate accretion can contribute approximately to a mild metal enhancement, but only for rapid migration (Fig. 5).

We found that the accretion contribution of highly refractory elements such as $\mathrm{Ca}$, for example, is small compared to those of I and V elements. In this case, what would be the meaning if, in case of a net metallic enhancement, we were to observe that the $\mathrm{R}$ elements have the same levels of abundances as $\mathrm{V}$ and I elements? We can indeed infer that $\mathrm{R}$ and I elements were originally more mixed in the disk as we thought. In other words, $\mathrm{R}$ elements are contained in I and maybe only the two zones $\mathrm{V}$ and I are the more realistic representatives ones.

We can interpret these results in another way. At the end of Sect. 4.1, we discussed some effects of the mixing processes during the primeval stages of a solar/stellar nebula. The detection of silicate bands in comets (Hanner et al. 1994) indicates that crystalline forms can exist in these outer disk bodies (Bouwman et al. 2000; Wooden et al. 2000). Since these crystals can only be formed at the relatively high disk temperatures existing near the star, it has been proposed that a large-scale mixing occurred during the early disk epochs, transporting material from the inner 
to the outer regions of the disk. Mechanisms such as turbulence and large-scale meridional flows in regions close to the disk midplane can act in this way. In contrast, inflow can exist above the midplane (Keller \& Gail 2004). Whether these mechanisms can be changed by the presence of magnetohydrodynamical turbulence was studied by Carballido et al. (2005). Independently, we have ultimately identified for our case, two different situations depending on the extent of these mixing processes. On the one hand, for a partial mixing we maintain the R, I, and V zones or only V and I. On the other hand, we obtain a single large intermediate zone disk when the early disk is completely and totally mixed.

\section{Results and conclusions}

In Fig. 8, we present one of our most important result: the relative distribution of the abundances of refractory, intermediate, and volatile elements as a function of their condensation temperatures for four CoRoT stars. A flat distribution is found for all of them, even if CoRoT-3 presents a slight tendency to display a positive slope. Clearly, a more reliable determination of this slope would be obtained if a complete collection of abundances of the highly volatile CNO elements was at hand. In any case, we also note the importance to dispose of measurements of the volatile $\mathrm{S}$ and $\mathrm{Zn}$ elements.

When considering the metallic abundances of CoRoT-2 and CoRoT-4, derived in this work, we found that the metallicity of CoRoT-4 is somewhat higher than that proposed by Bouchy et al. (2008), hence confirming a mild metallic excess. We compare the stellar parameters with those in the literature for CoRoT-2 and CoRoT-4 in Table 2.

CoRoT-2 star deserves particular attention because of its significant stellar activity and its youth indicators represented, respectively, by its $\mathrm{Ca}$ II H and $\mathrm{K}$ lines (Fig. 3) and its Li resonance line (Fig. 1), contrasting sharply with those of the older 1 Gyr star CoRoT-4 (Moutou et al. 2008), which does not exhibit these features. Its age can be determined from the equivalent width $(E W)$ and abundance of Li by using diagrams of the $\mathrm{Li}$ values as a function of the $T_{\text {eff }}$ values. By considering its measured $E W$ of $124 \mathrm{~m} \AA$ we found, using the diagrams presented by Torres et al. (2006, 2008), that this star falls in the lower dispersion limit of the $\mathrm{Li}$ abundances found for the members of the Pleiades cluster, with an age of $119 \pm 20 \mathrm{Myr}$ (Ortega et al. 2007). By using its Li abundance found here of 2.7, we arrived at the same position in the diagrams of Torres et al. (2006) and da Silva et al. (2009). From all these matches, we infer an age of 120 Myr for CoRoT-2, from which Bouchy et al. (2008) proposed an age of $<500 \mathrm{Myr}$. This new age implies that of CoRoT2 is one of the youngest known two stars with planets, the other being HD 70573, a debris-disk type star of a similar age and $\mathrm{Li}$ abundance (Setiawan et al. 2007). The $T_{\text {eff }}$ values of these two solar-type stars are in a short temperature interval, where old solar-type stars with planets exhibit a peculiar Li depletion with respect to similar stars without planets (Israelian et al. 2009). CoRoT-2 and HD 70573 are unaffected by this Li depletion due to their youth (see also Pinsonneault 2009).

We performed simulations of planetary migration into an internal disk composed of differentiated particles (planetesimals) with refractory, intermediate, and volatile properties, and applied these simulations to three CoRoT systems. The accreted material contains large and similar contributions of $I$ and $V$ particles and a very small contribution of pure refractory elements, as shown in Fig. 4. In other words, accretion is mainly "cool" and "warm" and not "hot" as largely mentioned in the literature. Because zone I exhibits a mixture of chemical properties, mainly containing common elements such as $\mathrm{Fe}$, both intermediate and volatile contributions produce a flat distribution of $[\mathrm{X} / \mathrm{H}]$ versus $T_{\mathrm{C}}$, as for the observed three CoRoT systems considered here.

When there is a complete primeval mixing of elements in the disk, a single intermediate zone will be maintained producing a flat distribution of abundances as a function of $T_{\mathrm{C}}$. However, if the mixing is incomplete or partial, some $\mathrm{R}$ and $\mathrm{V}$ rocky material will be maintained near and far from the star, respectively. An extended I zone (see top of Fig. 8) will control a large part of the slope. In that case, the agreement of the model with observations will depend mainly on the extended distribution of the element abundances with $T_{\mathrm{C}}<304 \mathrm{~K}$. Nevertheless, this region, containing volatile and highly volatile elements such as $\mathrm{CNO}$ and noble gases, in general, requires spectra of higher $S / N$ than our own to measure the abundances. By considering our $\mathrm{O}$ abundances for CoRoT- 2 and 4 and the $\mathrm{C}$ abundances for CoRoT- 3 by Deleuil et al. (2008) and that for CoRoT-5 (Rauer et al. 2009), we obtained one collection of quite flat gradients as presented in Fig. 8. We note that CoRoT-3, for which the gradient is the least flat, is a peculiar case because its "planet" is probably a brown dwarf star. We conclude that to obtain a reliable collection of highly volatile element abundances of $\mathrm{CNO}$ and noble gases, data of even higher $S / N$ are required.

In any case, independently of whether accretion is important or not, depending on the accreting mass, the main result of this work, is that, for the CoRoT systems considered, flat distributions that appear to be observationally the rule, do not represent the absence of a self-enrichment mechanism as sometimes mentioned in the literature. In contrast, we show here that flat distributions of the elements abundances as function of $T_{\mathrm{C}}$ could be a natural result of accretion.

Does the discussed type of migration represent true situations for the CoRoT systems 2, 3, and 4? This is not the case for, at least systems 2 and 3, where unrealistically high disk masses are necessary to bring the planets to their observed final distances with respect to the star (Adams \& Laughlin 2003). System 4 can be considered an exception. A different migration of type II in a disk containing gas (Papaloizou \& Terquem 2006; Armitage 2007) has to be invoked for CoRoT-2. The CoRoT-3 system is particularly difficult and could represent a challenge to migration theories. If this planetary body, with an exceptionally high mass of $21.66 M_{\mathrm{J}}$ is a real planet and not a brown dwarf, a type II migration study must first avoid the collision of the planet with the star due to the large planetary eccentric orbits developed, which is the case, at least, for a $\sim 10 M_{\mathrm{J}}$ (Rice et al. 2008). These authors mention that if the gas disk dissipates quickly, the eccentric orbits of these massive planets could eventually be tidally circularized (Ford \& Rasio 2006). Is this the case of CoRoT-3 system?

CoRoT-4 system with a planet of $0.72 M_{\mathrm{J}}$, also requires a high primeval disk mass of $\sim 0.2-0.3 M_{\odot}$. Even if it were an extreme case, it could be acceptable for a star of $1.2 M_{\odot}$. Nevertheless, our simulations indicate that in this case only a very rapid migration of $1000 \mathrm{yr}$ can produce a metal enhancement as observed of $[\mathrm{I} / \mathrm{H}] \sim 0.1$, if occurred in the first 20 $30 \mathrm{Myr}$, when the stellar convective layer for the first time attains its minimum configuration (Ford et al. 1999), This could also be possible in principle, even for the smaller planet of mass $0.467 M_{\mathrm{J}}$ related to CoRoT-5 (Rauer et al. 2009). We also show that these results are highly dependent on the model adopted for the disk distribution regions in terms of refractory, intermediate, and volatile elements and the other parameters considered. 
Acknowledgements. Part of this work was supported by CAPES, CNPq and FAPESP. The authors would like to thank the referee for suggestions that improved the paper.

\section{References}

Adams, F. C., \& Laughlin, G. 2003, Icarus, 163, 290

Aigrain, S., Collier Cameron, A., Ollivier, M., et al. 2008, A\&A, 488, L43

Alonso, R., Auvergne, M., Baglin, A., et al. 2008, A\&A, 482, L21

Ammler-von Eiff, M., Santos, N. C., Sousa, S. G., et al. 2009, A\&A, 507, 523

Anders, E., \& Grevesse, N. 1989, Geochim. Cosmochim. Acta, 53, 197

Armitage, P. J. 2007, ApJ, 665, 1381

Boesgaard, A. M., Armengaud, E., King, J. R., Deliyannis, C. P., \& Stephens, A. 2004, ApJ, 613, 1202

Bouchy, F., Queloz, D., Deleuil, M., et al. 2008, A\&A, 482, L25

Bouwman, J., de Koter, A., van den Ancker, M. E., \& Waters, L. B. F. M. 2000, A\&A, 360, 213

Bruntt, H., De Cat, P., \& Aerts, C. 2008, A\&A, 478, 487

Carballido, A., Stone, J. M., \& Pringle, J. E. 2005, MNRAS, 358, 1055

Cayrel de Strobel, G., \& Spite, M. 1988, The impact of very high S/N spectroscopy on stellar physics: Proceedings of the 132nd Symposium of the International Astronomical Union held in Paris, France, June 29-July 3, 1987, IAU Symp., 132

Cochran, W. D., Endl, M., Wittenmyer, R. A., \& Bean, J. L. 2007, ApJ, 665, 1407

da Silva, L., Torres, C., de La Reza, R., et al. 2009, A\&A, 508, 833

Davis, S. S. 2005, ApJ, 620, 994

Deleuil, M., Deeg, H. J., Alonso, R., et al. 2008, A\&A, 491, 889

Ecuvillon, A., Israelian, G., Santos, N. C., Mayor, M., \& Gilli, G. 2006, A\&A, 449,809

Ford, E. B., \& Rasio, F. A. 2006, ApJ, 638, L45

Ford, E. B., Rasio, F. A., \& Sills, A. 1999, ApJ, 514, 411

Gonzalez, G. 1997, MNRAS, 285, 403

Gonzalez, G. 2003, Rev. Mod. Phys., 75, 101

Gonzalez, G. 2006a, MNRAS, 367, L37

Gonzalez, G. 2006b, PASP, 118, 1494

Hanner, M. S., Hackwell, J. A., Russell, R. W., \& Lynch, D. K. 1994, Icarus, 112,490

Hobbs, L. M., Thorburn, J. A., \& Rebull, L. M. 1999, ApJ, 523, 797

Israelian, G., Delgado Mena, E., Santos, N. C., et al. 2009, Nature, 462, 189

Kaufer, A., Stahl, O., Tubbesing, S., et al. 1999, The Messenger, 95, 8

Keller, C., \& Gail, H.-P. 2004, A\&A, 415, 1177

Kupka, F., Piskunov, N., Ryabchikova, T. A., Stempels, H. C., \& Weiss, W. W. 1999, A\&AS, 138, 119
Kurucz, R. L. 1993, VizieR Online Data Catalog, 6039, 0

Lambert, D. L., Heath, J. E., Lemke, M., \& Drake, J. 1996, ApJS, 103, 183

Levison, H. F., \& Duncan, M. J. 1994, Icarus, 108, 18

Lissauer, J. J. 1993, ARA\&A, 31, 129

Lodders, K. 2003, ApJ, 591, 1220

Moutou, C., Bruntt, H., Guillot, T., et al. 2008, A\&A, 488, L47

Murray, N., Hansen, B., Holman, M., \& Tremaine, S. 1998, Science, 279, 69

Ortega, V. G., Jilinski, E., de La Reza, R., \& Bazzanella, B. 2007, MNRAS, 377, 441

Papaloizou, J. C. B., \& Terquem, C. 2006, Reports on Progress in Physics, 69, 119

Pasquini, L., Döllinger, M. P., Weiss, A., et al. 2007, A\&A, 473, 979

Pinsonneault, M. 2009, Nature, 462, 168

Piskunov, N. E., Kupka, F., Ryabchikova, T. A., Weiss, W. W., \& Jeffery, C. S. 1995, A\&AS, 112, 525

Quillen, A. C. 2006, MNRAS, 372, L14

Rauer, H., Queloz, D., Csizmandia, S., et al. 2009, A\&A, 506, 281

Rice, W. K. M., Armitage, P. J., \& Hogg, D. F. 2008, MNRAS, 384, 1242

Sadakane, K., Ohkubo, M., Takeda, Y., et al. 2002, PASJ, 54, 911

Santos, N. C., Israelian, G., \& Mayor, M. 2001, A\&A, 373, 1019

Santos, N. C., Israelian, G., \& Mayor, M. 2004, A\&A, 415, 1153

Setiawan, J., Weise, P., Henning, T., et al. 2007, ApJ, 660, L145

Shen, Z.-X., Liu, X.-W., Zhang, H.-W., Jones, B., \& Lin, D. N. C. 2007, ApJ, 660,712

Smith, V. V., Lambert, D. L., \& Nissen, P. E. 1998, ApJ, 506, 405

Smith, V. V., Cunha, K., \& Lazzaro, D. 2001, AJ, 121, 3207

Sneden, C. A. 1973, Ph.D. Thesis, AA (The University of Texas at Austin)

Takeda, Y. 2003, A\&A, 402, 343

Takeda, Y., Sato, B., Kambe, E., et al. 2001, PASJ, 53, 1211

Torres, C. A. O., Quast, G. R., da Silva, L., et al. 2006, A\&A, 460, 695

Torres, C. A. O., Quast, G. R., Melo, C. H. F., \& Sterzik, M. F. 2008, The Southern Sky ASP Monograph Publications, 5, 757

Wehrstedt, M., \& Gail, H.-P. 2002, A\&A, 385, 181

Wiese, W. L., Smith, M. W., \& Miles, B. M. 1969, Atomic transition probabilities, Sodium through Calcium, A critical data compilation (NSRDS-NBS, Washington, D.C.: US Department of Commerce, National Bureau of Standards), 2

Winter, O. C., de La Reza, R., Domingos, R. C., Boldrin, L. A. G., \& Chavero, C. 2007, MNRAS, 378, 1418

Wisdom, J. 1980, AJ, 85, 1122

Wisdom, J., \& Holman, M. 1991, AJ, 102, 1528

Wooden, D. H., Butner, H. M., Harker, D. E., \& Woodward, C. E. 2000, Icarus, 143,126 